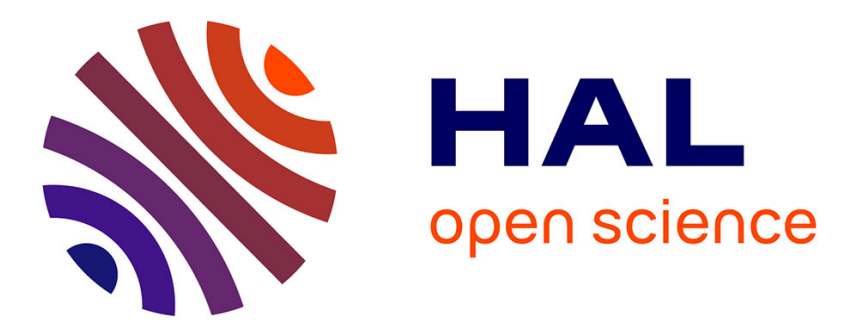

\title{
Pour en finir avec la " cité hydraulique"? Note de lecture de l'ouvrage de Didier Pillot, Jardins et rizières du Cambodge. Les enjeux du développement agricole, 2007
}

Éric Bourdonneau

\section{To cite this version:}

Éric Bourdonneau. Pour en finir avec la " cité hydraulique "? Note de lecture de l'ouvrage de Didier Pillot, Jardins et rizières du Cambodge. Les enjeux du développement agricole, 2007. Bulletin de l'Ecole française d'Extrême-Orient, 2010, pp.409-437. 10.3406/befeo.2010.6145 . halshs-02530979

\section{HAL Id: halshs-02530979 \\ https://shs.hal.science/halshs-02530979}

Submitted on 14 Apr 2020

HAL is a multi-disciplinary open access archive for the deposit and dissemination of scientific research documents, whether they are published or not. The documents may come from teaching and research institutions in France or abroad, or from public or private research centers.
L'archive ouverte pluridisciplinaire HAL, est destinée au dépôt et à la diffusion de documents scientifiques de niveau recherche, publiés ou non, émanant des établissements d'enseignement et de recherche français ou étrangers, des laboratoires publics ou privés. 


\section{Pour en finir avec la " cité hydraulique » ? Note de lecture de} l'ouvrage de Didier Pillot, Jardins et rizières du Cambodge. Les enjeux du développement agricole, 2007

Éric Bourdonneau

\section{Citer ce document / Cite this document :}

Bourdonneau Éric. Pour en finir avec la « cité hydraulique » ? Note de lecture de l'ouvrage de Didier Pillot, Jardins et rizières du Cambodge. Les enjeux du développement agricole, 2007. In: Bulletin de l'Ecole française d'Extrême-Orient. Tome 97-98, 2010. pp. 409-437;

doi : https://doi.org/10.3406/befeo.2010.6145

https://www.persee.fr/doc/befeo_0336-1519_2010_num_97_1_6145

Fichier pdf généré le 08/11/2019 


\title{
Résumé
}

L'ouvrage de Didier Pillot vise avant tout à définir les enjeux du développement agricole pour le Cambodge contemporain. Cependant l'auteur privilégie tout au long de son étude une approche dynamique des pratiques agraires et son propos inclut de longs développements sur les époques plus anciennes. La présente note de lecture entend donc souligner l'intérêt de cet ouvrage pour la compréhension de l'histoire agraire du Cambodge, tout en anticipant certaines des objections qu'il pourrait susciter aux yeux des archéologues. Elle se propose d'identifier les grandes hypothèses qui sous-tendent l'analyse de Didier Pillot, mais aussi d'imprimer certaines inflexions à l'histoire sur la longue durée qui est esquissée par l'auteur, en espérant définir là les termes d'un dialogue nécessaire entre historiens et agronomes.

\begin{abstract}
The main purpose of Didier Pillot's work is to define what is at stake in the agricultural development of modern Cambodia. However the author favors throughout his study a dynamic approach of the agrarian practices and his argument includes long developments on historical times. The present review article thus intends to underline the interest of this work for the knowledge of the agrarian history of Cambodia, while anticipating some of the objections that could be made by archaeologists. It aims at identifying the main hypotheses that underlie Didier Pillot's analysis, but also at partly reorienting the long term history outlined by the author. It hopes to open up a necessary dialogue between historians and agronomists.
\end{abstract}




\section{Pour en finir avec la « cité hydraulique »?}

Note de lecture de l'ouvrage de Didier Pillot, Jardins et rizières du Cambodge. Les enjeux du développement agricole, Paris/Nogent-sur-Marne, Karthala/Gret, 2007.

\section{Éric BourdonNEAU*}

« Gigantisme trompeur :

il est beaucoup moins coûteux d'édifier ce qu'archéologues et touristes appellent une haute culture riche en monuments, que de faire manger une population à peu près à sa faim »

Veyne $1976: 142$

Les auteurs des premiers inventaires archéologiques trouvaient tout naturel d'accoler les initiales du « découvreur » aux numéros d'inventaire créés. La pratique est révolue mais l'archéologue reste homme de terrain et, comme il arrive parfois, le terrain se transforme, aux yeux dudit archéologue, en «son territoire ".

À distance a priori d'un tel territoire, le présent ouvrage s'adresse avant tout aux professionnels du secteur rural soucieux des enjeux du développement agricole du Cambodge contemporain. Au regard des ouvrages similaires, il présente cependant la particularité d'inclure un long chapitre, sur les six qui le composent, consacré à l'histoire de l'agriculture du pays « à travers les âges » avec, comme on s'y attend, une longue partie réservée à l'examen du concept de "cité hydraulique » formulé par Bernard Philippe Groslier. Après quelques autres, Didier Pillot, qui est agronome et n'est donc pas moins homme de terrain, prend ainsi le temps de parcourir un territoire revendiqué, parfois avec force, par les archéologues.

La démarche n'est pas sans risque. Elle est également source de malentendus innombrables. La présente note vise néanmoins à en souligner l'intérêt pour l'historien, anticipant certaines des objections qu'elle pourrait susciter chez le lecteur pressé - et de l'archéologue soucieux de la maîtrise de son territoire - et qui justifieraient de le dispenser d'une lecture plus approfondie de l'ouvrage.

La démarche de l'auteur est risquée parce qu'il n'est pas chose aisée de prendre la mesure d'une production scientifique - en particulier dans ses évolutions les plus récentes sans en être l'un des acteurs à part entière. Cela est sans doute encore plus vrai au sein d'un espace de recherche aussi peu structuré que peuvent l'être les études sur le

* Éric Bourdonneau est maître de conférences à l'École française d’Extrême-Orient. 
Cambodge ancien. Et c'est donc un peu sans surprise que l'on découvre une bibliographie très lacunaire pour les périodes anciennes. Aucun des travaux menés depuis une dizaine d'années aussi bien sur la région d'Angkor que sur le delta du Mékong (côtés cambodgien et vietnamien de la frontière) n'est mentionné. Il est vrai qu'il s'agit encore, pour une large part, de recherches en cours. Les travaux publiés demeurent assez peu nombreux et n'ont débouché, pour l'heure, sur aucun ouvrage majeur. La gestation assez longue du livre de Pillot explique sans doute aussi ces lacunes bibliographiques. Sa publication marque l'aboutissement de travaux et d'enquêtes de terrain engagés de longue date par le Gret (Groupe de recherche et d'échanges technologiques) au Cambodge, remontant au début des années 1990.

Cette approche au plus près de la réalité du terrain, où la recherche agronomique se conjugue à la proximité avec les paysans, explique peut-être également la relative pauvreté de la documentation cartographique de l'ouvrage. Les trois vignettes d'images satellites reproduites dans le cahier central des illustrations compensent difficilement une telle pauvreté '. À l'heure de l'usage généralisé des systèmes d'informations géographiques (SIG), cela rebutera certainement plus d'un lecteur-archéologue, ce dernier étant de plus en plus accoutumé à ce type d'outil informatique, riche en potentialités cartographiques. "Montre-moi ta carte et je dirai qui tu es!", pourrait-on faire valoir à la suite des géographes. $\Lambda u$ sein de la littérature abordant la thématique de la "cité hydraulique » et l'histoire agraire du Cambodge ancien, les cartes publiées, ou ce qui en tient lieu, apparaissent souvent à l'image des approches adoptées.

On pourra encore regretter le traitement réservé à certains des épisodes ou à des processus majeurs de l'histoire ancienne du pays khmer tels, par exemple, que l'indianisation ou la transition Funan-Zhenla. Les survols proposés, comme les références ponctuelles faites aux sources ne sont pas toujours bien maîtrisés, sans que l'on ait trop à s'en étonner (un exemple parmi d'autres [Jardins et rizières du Cambodge, noté ci-dessous JRC: 60] : la plus ancienne inscription en vieux khmer portant une date ne rapporte en aucun cas les épisodes de la conquête du Funan par le Zhenla).

Les historiens et les archéologues auraient tort, nous semble-t-il, de trop s'arrêter aux faiblesses de ce chapitre historique. Il comporte, comme nous allons le voir, quelques pages décisives qui méritent d'autant plus leur attention qu'elles soulignent inversement les faiblesses des propos tenus par les archéologues en matière d'agronomie.

Par ailleurs, il s'agit précisément d'un chapitre qui, en tant que tel, n'est pas à lire de façon indépendante du reste de l'ouvrage. Il nous semble qu'une bien mauvaise lecture de ce dernier serait de le scinder, séparant, d'un côté, les chapitres supposés relever, à proprement parler, du savoir de l'agronome et, de l'autre, un essai d'histoire qui serait perçu comme une excursion juxtaposée artificiellement et plus ou moins légitime. Si certaines digressions de ce chapitre historique (chap. 2) - ainsi celles consacrées à l'essor et au déclin d'Angkor évoquant, à l'image de la littérature existante, les guerres, le commerce, la religion - alimentent en partie ce type de lecture, les nombreux renvois au reste de l'ouvrage intègrent néanmoins clairement celui-ci à la réflexion plus globale développée par l'auteur. Il y a là une précaution qui, peut-on espérer, dispensera Pillot des critiques rapides dont certains de ces devanciers non archéologues, ayant pris part

1. Sur le plan formel, outre quelques coquilles (ainsi «Pallana » pour Pallava, «Daggens » pour Dagens), on pourra également regretter que les références bibliographiques utilisées ne soient pas toujours mentionnées ou qu'elles le soient souvent sans indication de pagination. 
au débat sur la " cité hydraulique », ont pu faire l'objet en publiant leurs contributions dans des revues d'archéologie.

Les historiens et les archéologues trouveront d'autant plus d'intérêt à ne pas se limiter à la seule partie les concernant a priori, que l'ensemble de l'ouvrage porte une attention soutenue aux multiples facteurs de changements des pratiques agraires. Bien au-delà du chapitre historique, qui fait suite à la présentation du « milieu naturel » (chap. 1), le lecteur retrouve ce souci de l'auteur d'aborder ces pratiques dans une perspective dynamique, selon le principe - auquel l'historien ne saurait être insensible - que l'on ne voit jamais aussi bien les choses que lorsqu'elles bougent. Dans la perspective de l'ouvrage, il s'agit avant tout pour l'auteur de s'interroger sur le potentiel de développement de l'agriculture à l'aube du $\mathrm{XXI}^{\mathrm{e}}$ siècle. Ces réflexions se développent en particulier dans le chapitre final («L'art et la manière », chap. 6) envisageant les scénarios possibles de transformation de l'agriculture pour les prochaines décennies.

Ces réflexions tournées vers l'avenir encadrent, avec le chapitre historique, la description des pratiques actuelles qui occupent les trois chapitres placés au cœur de l'ouvrage : le premier d'entre eux («Hommes, terres et eau : les conditions de production », chap. 3) aborde les grands traits de la production agricole, dans ses aspects à la fois sociaux et économiques, et insiste sur le problème majeur de l'accès à la terre. Le chapitre suivant («Les systèmes de base de riziculture », chap. 4) détaille les principales techniques de riziculture, en soulignant la diversité des stratégies mises en œuvre par les paysans, selon les conditions environnementales locales, mais aussi les facteurs économiques et sociaux esquissés au chapitre précédent. Enfin, un dernier chapitre réserve une analyse spécifique à la culture des camkâr, à laquelle fait écho la mention des « jardins » dans le titre de l'ouvrage ( La polyculture des chamcars », chap. 5). Une caractéristique commune de ces chapitres demeure, là encore, l'intérêt porté à l'évolution des pratiques, l'auteur faisant l'inventaire des similitudes et des différences entre les transformations observées depuis le début des années 1990 et l'agriculture, qualifiée un peu rapidement de «traditionnelle », antérieure aux événements tragiques des années 1970.

Il s'agit donc d'un ouvrage ambitieux dont il n'est guère question de discuter tous les aspects. Nous nous limiterons dans ce qui suit à quelques-uns des points qui nous paraissent susceptibles d'alimenter les réflexions de l'historien, bien au-delà, nous l'avons dit, des seules remarques contenues dans le chapitre historique. La démarche ne vise pas tant à extraire la " partie utile " de l'ouvrage, qu'à reconnaître les grands axes d'une vision d'ensemble sur l'agriculture du Cambodge, et de proposer certaines inflexions parmi celles que l'historien est amené inévitablement à imprimer à une telle vision, selon sa propre maîtrise des sources. Cela consiste pour une part à en moduler différemment l'argumentation, quitte à faire jouer les unes contre les autres certaines observations. Il ne s'agit ni de tourner le dos aux travaux des agronomes, ni d'en reproduire les conclusions telles quelles, mais bien de définir, espère-t-on, les termes d'un dialogue et, au vu du travail qu'il reste à accomplir, d'esquisser ce qui pourrait être un programme de recherche en ce domaine. 


\section{Le doute sur la « cité hydraulique»}

En raison de son caractère emblématique, et de ses nombreuses implications, on commencera par le traitement réservé à la thèse de la " cité hydraulique " et l'on se concentrera d'emblée sur les remarques les plus décisives à nos yeux.

Au cœur du concept de la " cité hydraulique », au travers des différentes formulations qu'en a données Groslier, se trouvent les haray d'Angkor ${ }^{2}$, définis comme d'immenses lacs artificiels visant à emmagasiner l'eau dans le but d' « assurer une répartition optimale de l'eau pour cultiver le riz » grâce à un système de canaux d'irrigation. Lors des premières formulations de la thèse ${ }^{3}$, Groslier affichait une belle assurance dans la reconstitution de ce réseau : les rizières, écrivait-il, «étaient irriguées par un réseau toujours visible, et que nous avons relevé, de collecteurs et d'artérioles, qui distribuait jusque dans les moindres parcelles l'eau des rivières et des baray " (Groslier 1958: 110-111). Une vingtaine d'années plus tard, le constat n'est plus le même : "Les canaux d'irrigation proprement dits étaient rares : ils étaient pratiquement inutiles grâce à [un] dispositif ingénieux » (Groslier 1979 : 168). Groslier restitue ici l'existence d'un canal périphérique extérieur courant le long de la digue aval (la terre nécessaire aux digues des baray étant prélevée de part et d'autre de leurs tracés). En même temps que ce canal aurait permis de collecter les eaux pouvant s'infiltrer sous les digues, une brèche dans celles-ci aurait permis de le rcmplir en eau. À partir de là, « les rizières étaient alimentées directement, l'eau passant d'un casier à l'autre selon la pente » (Groslier 1979: 168). Ce dispositif serait simplement complété, à l'occasion, par un canal qui déporterait latéralement la nappe d'eau de façon à étendre la zone des rizières irriguées.

En dehors de ces deux types de canaux (canal périphérique et canal latéral), aucun autre ouvrage de distribution, acheminant l'eau jusque dans les rizières en aval, ne serait donc nécessaire et n'a été cartographié par Groslier (contrairement donc à ce que lui-même affirmait quelques années plus tôt). L'absence d'un tel système de distribution représentait, aux yeux du principal contradicteur de Groslier, van Liere (1980), un obstacle majeur à la thèse de la " cité hydraulique ", mais ce dernier ne s'attardait guère sur l'hypothèse proposée alors par Groslier d'une irrigation par gravité. La critique de Pillot se fait, sur ce point, beaucoup plus précise.

Évoquant le dispositif ainsi imaginé par Groslier - l'eau étant supposée s'acheminer spontanément de parcelle à parcelle -, Pillot écrit : «cela est totalement impossible, surtout s'il ne s'agit que d'irrigation d'appoint, visant à compenser un manque de pluie. En effet, les besoins en irrigation d'appoint sont par nature variables, en termes de date et de quantité, selon les parcelles, leur taille, la nature plus ou moins filtrante de leur sol, la date des semis, etc. Il est rigoureusement impossible dans ces conditions qu'un agriculteur soit dépendant, pour le volume d'eau dont il a besoin à une date précise, de la nature des besoins du voisin dont la parcelle jouxte la sienne à son amont immédiat. De tels systèmes d'irrigation de parcelle à parcelle n'existent dans le monde que dans des situations où l'eau est disponible en abondance et où l'on peut maintenir une circulation partout dans les parcelles. Cette circulation d'eau doit pouvoir s'opérer même en dehors des moments de besoin d'irrigation d'une parcelle particulière, de telle sorte que les agri-

2. Il faudrait, en réalité, être plus précis : tous les barạ n’illustrent pas les principes de la «cité hydraulique " définis par Groslier, celui-ci reconnaissant que le bassin du temple de Preah Khan n’a guère pu remplir une fonction d'irrigation semblable à celle attribuće aux deux autres grands harca! de la plaine d'Angkor (le baral occidental et le haral oriental).

3. Sur la genèse et l'historique de la thèse de la " cité hydraulique ». cf. Fletcher et al. 2008 a. 
culteurs en contrebas aient toujours un accès à l'eau. À Angkor, on est évidemment très loin d'une telle configuration, sauf en saison des pluies! " (JRC: 73). La topographie de la plaine d'Angkor, poursuit Pillot, est tout autant un obstacle au "dispositif ingénieux » restitué par Groslier : "l'eau ne peut circuler de parcelle à parcelle que si la pente est suffisante et régulière. Or entre les baray d'Angkor et la rive archéologique du Lac, il y a dix kilomètres..., mais seulement huit mètres de dénivelé, soit une pente de 0,8 pour mille. En aucun cas une si faible pente ne peut permettre ce type de circulation d'eau ».

Une longue citation de l'ouvrage ne nous semblait pas, sur ce point, inutile. L'apport de Pillot est d'envisager ici concrètement, d'un point de vue technique, la question essentielle de la maîtrise de l'eau au sein des rizières. Elle vise ce qui est demeuré l'une des principales zones d'ombre dans le raisonnement de Groslier. On peut d'ailleurs s'étonner du relatif silence de la littérature sur ce sujet, qui est symptomatique du mode encore très allusif des points de vue exprimés jusqu'à présent. Pillot lui-même, pourrait-on faire remarquer, ne place pas une telle objection au cœur de son argumentation; elle figure simplement comme l'une des objections soulevées par l'auteur, parmi de nombreuses autres qui n'ont pas toutes, à nos yeux, la même pertinence.

Encore faut-il s'assurer qu'aucun système de distribution n'a effectivement existé. Pillot ignore sur ce point les récents développements de la littérature qui s'est, pour une part, orientée vers une réhabilitation de la thèse de la «cité hydraulique » en soutenant, en réponse aux critiques de van Liere, que les baray avaient bel et bien une fonction d'irrigation des rizières situées en aval ${ }^{4}$. Ce nouveau tournant de l'historiographie trouve son point de départ dans la lecture des formes du paysage au sud-ouest du baray occidental. L'analyse proposée réinterprète comme la partie centrale d'un ancien système de canaux d'irrigation certains éléments déjà repérés par Groslier et identifiés par celui-ci comme les douves de l'ancienne ville de «Banteay Chhoeu » (dans l'angle sud-ouest du baray).

Nous avons évoqué ailleurs quelques-unes des raisons pour lesquelles l'interprétation de Groslier sur ce point devait, selon nous, être maintenue ${ }^{5}$. Le constat de l'absence d'un système de distribution de l'eau jusqu'aux rizières demeure donc valide à nos yeux, comme la critique de l'hypothèse d'une irrigation en aval des baray. Or il s'agit bien là du cour de la thèse de la " cité hydraulique ${ }^{6}$.

Faut-il renoncer définitivement à celle-ci ? C'est évidemment la thèse privilégiée ici, puisque nous venons de voir que nous ne saurions désormais retenir l'hypothèse d'une circulation de l'eau de parcelle à parcelle comme l'avait imaginée Groslier. Cependant

4. Il s'agit essentiellement des travaux du Greater Angkor Project, dirigé par Roland Fletcher et Christophe Pottier (par ex. Fletcher et al. 2008b).

5. Cf. Pottier 2000: 109-112 et fig. 1 et 2 et la note critique que nous y consacrons in Bourdonneau $2007: 137$, n. 62.

6. La formulation de cette thèse par Groslier inclut de nombreux autres aspects et s'étend virtuellement à l'ensemble de la gestion de l'eau dans la région d'Angkor. Celui-ci insiste sur le rôle important de ce qu`il désigne comme la "banlieue hydraulique » et consacre beaucoup de temps à la description des douves des temples et cités. Il n'est pas inutile de le rappeler, mais on ne saurait "sauver » ainsi la "cité hydraulique ». Pour la clarté des débats, aucune tentative de réhabilitation de la «cité hydraulique » en tant que telle ne saurait exclure la thèse de fonction agricole de ces mêmes baray au bénéfice de superficies proportionnées à leurs dimensions hors normes. Et, de ce point de vue, que l'on crédite les barcu' d'une double ou d'une triple récolte de riz, ou encore d'une récolte unique (mais qui serait garantie par les réserves d'eau stockée, y compris lors des années difficiles), ne change pas grand chose : il s'agit bien là de différentes formulations de la thèse dite de la « cité hydraulique », ayant toutes en commun d'attribuer aux barạ. un rôle essentiel dans l'essor et/ou la viabilité économique d'Angkor. 
on conviendra que les discussions sur l'interprétation des formes du paysage au sud des baray n'ont pas encore, à ce stade, été menées jusqu'à leur terme. À défaut donc d'en finir avec la "cité hydraulique ", le doute est néanmoins permis et c'est là plus qu'il n'est nécessaire pour que l'on soit fondé à poursuivre la lecture de l'ouvrage de Pillot.

\section{Au-delà des baray, les termes du débat}

\section{Les "deux sens du mot culture " ${ }^{7}$}

La thèse de la " cité hydraulique " étant ainsi (provisoirement) écartée, il s'agit à présent d'examiner les solutions alternatives proposées par Pillot pour définir les grandes caractéristiques de la production agricole et l'éventuel essor de celle-ci à l'époque angkorienne. Après van Liere et bien d'autres, l'auteur attire l'attention sur la culture du riz de décrue. Si la thèse n'est pas nouvelle, le modèle esquissé est un peu plus complexe que ce qui a pu être proposé jusqu'à maintenant.

L'auteur souligne tout d'abord le rôle du riz flottant : «Parmi tous les systèmes de culture du riz, c'est celui qui, dès lors que l'on dispose de surfaces suffisamment vastes, permet la meilleure productivité du travail, c'est-à-dire la production nécessaire à l'entretien de sa famille avec le minimum d'efforts " (JRC: 77). Or, poursuit Pillot, c'est précisément un atout de la région bordant la rive nord du Tonle Sap : de larges zones sont favorables à la culture du riz flottant pendant la crue du lac. Les paysans disposant de temps et de terres disponibles bien au-delà du seuil nécessaire à leur subsistance, le dégagement de premiers surplus est tout à fait envisageable.

Il s'agit néanmoins, observe ailleurs Pillot, d'un système de culture aléatoire, et c'est davantage le riz de décrue, cultivé de façon intensive sur de petites surfaces, qui permettrait de dégager des surplus (dès lors que la pression démographique s'élève). Celui-ci étant repiqué au fur et à mesure du retrait des eaux, et donc sur des terres qui viennent d'être recouvertes de limon par la crue, « sa culture intervient au moment de l'année où l'ensoleillement est optimal $»(J R C: 78)$. S'il est très exigeant en travail à la différence du riz flottant, le riz de décrue est aussi beaucoup plus productif à l'unité de surface.

Pillot n'oublie pas la contribution des zones de terrasses, mais celle-ci n'est guère formulée en termes de surplus. L'auteur esquisse ici une vision assez sombre : «Avec un rendement qui ne pouvait guère excéder une tonne par hectare et une superficie par famille qui, pas plus qu'aujourd'hui, ne peut guère dépasser un hectare, à cause des contraintes de temps de travail au moment des repiquages et des désherbages, leur production suffit tout juste à nourrir ceux qui les travaillent » $(J R C: 80)$. Sur les terres situées le long du lac, Pillot suggère que les paysans combinaient riziculture de mousson (des terrasses) et riziculture de décrue, les calendriers de travaux de l'une et de l'autre étant complémentaires. C'est cette agriculture (à laquelle viendrait s'ajouter la culture du riz flottant) qui, soutient l'auteur, aurait « nourri » les cités angkoriennes. Sur les terres plus éloignées de la ligne de crue, où une telle combinaison n'est pas possible, la contribution des paysans s'estimerait non pas en paniers de riz mais en nombre de bras libérés pour des activités non agricoles, essentiellement lors de la période creuse du calendrier agraire, correspondant à la saison sèche : «c'est cette seconde agriculture qui aurait alors "construit" les cités angkoriennes » $(J R C: 81)$.

7. Nous empruntons la formule à Pierre Bonnassié, historien de l'occident médiéval (Bonnassié 2001 [1996]). 
Deux grandes thèses sous-tendent le modèle proposé par Pillot.

L'une relève de la périodisation de l'histoire agraire du Cambodge. La dernière " révolution verte » remonterait à l'époque du Funan. L'époque angkorienne n'apporterait en elle-même aucune innovation majeure sur le plan technique. Sa force serait de trouver les ressorts d'une mobilisation inédite de la main-d'œuvre et de tirer profit de la situation particulière de la plaine de Siem Reap. Aux confins de la zone de crue et de décrue des eaux du Grand Lac, entourée par les terrasses, celle-ci permettrait de conjuguer harmonieusement les trois agricultures que les Khmers maîtrisaient dès l'époque préangkorienne : l'agriculture de riz flottant, l'agriculture du riz de décrue et la riziculture de terrasse, complétées par le maraîchage et les cultures « de berge ».

Tous les éléments des systèmes agricoles « traditionnels » seraient ainsi déjà en place au moment où les rois khmers implantent leurs capitales dans la plaine de Siem Reap, et tels que la colonisation les trouvera dix siècles plus tard : la culture attelée et ses outils, la charrue, la herse et la charrette, les instruments d'exhaure et de remontée de l'eau, etc.

L'autre grande thèse soutenue par Pillot est que l'agriculture de l'époque angkorienne serait « fondée avant tout, non pas sur l'artificialisation du milieu, mais sur l'utilisation des éléments naturels », à savoir la pluie de mousson et surtout la crue du lac : l'erreur des archéologues aurait été ici de s'être focalisés dix kilomètres «trop haut », sur la zone de terrasses, là même où étaient implantées les capitales successives, beaucoup moins productives que la zone de battement de la crue, sauf précisément à imaginer une transformation du paysage d'autant plus poussée, et improbable, que les terres ne se prêtaient guère au dégagement des surplus recherchés $(J R C: 76)$.

La deuxième de ces deux thèses retiendra d'abord notre attention. L'opposition entre deux agricultures, l'une fondée sur l'artificialisation du milieu, l'autre sur l'utilisation des éléments naturels, éclaire en des termes assez justes le débat mené sur l'agriculture angkorienne. Elle offre, ce faisant, l'intérêt d'une formulation qui met en perspective des travaux parfois étroitement bornés aux seuls aspects techniques du débat et rappelle, si nécessaire, que les techniques sont solidaires d'une vision du monde - une vision des rapports des hommes entre eux et au monde qui est le leur.

L'artificialisation du milieu est bien l'idée directrice qui sous-tend la thèse de la cité hydraulique. Les anciens Khmers, est-il supposé, intervenaient sur leur environnement pour pallier les insuffisances de la nature nourricière. L'irrégularité des pluies et la forte disparité entre la saison des pluies et la saison sèche sont ici pointées du doigt. Stocker l'eau, à une vaste échelle pour la distribuer à une vaste échelle, serait une nécessité. Il s'agit bien alors de dominer la nature, d'une anthropisation exacerbée contre celle-ci ou, plutôt, en dépit de celle-ci : en dépit des très faibles pentes, en dépit de sols sableux, en dépit de l'évaporation, etc.

Est-ce pousser la critique trop loin que de suggérer qu'en ce domaine, Groslier était peut-être trop homme de son temps ? Comme nous le fait observer Stéphane Boulakia ${ }^{8}$, il existe une remarquable relation de contemporanéité entre la formulation de la " cité hydraulique » et la "révolution verte » des décennies d'après-guerre marquées par un modernisme triomphant. Et il n'est sans doute pas interdit de voir un même fil rouge traverser la production scientifique et l'activité alors prolifique de Groslier : un interventionnisme certain dans le renouvellement des méthodes de restauration des temples, une

8. Agronome, responsable de la mission du CIRAD (Centre de coopération internationale en recherche agronomique pour le développement) au Cambodge. 
rigueur toute géométrique dans la stricte application des techniques de fouilles théorisées par Mortimer Wheeler font ici écho à l'accent mis sur les formidables progrès techniques comme explication de l'essor exceptionnel de l'empire khmer.

La thèse de la cité hydraulique invitait fortement à mesurer le niveau de civilisation de la société khmère d'après le degré d'anthropisation et de domination de la nature. $\mathrm{Au}$ Cambodge comme ailleurs, le raisonnement a séduit plus d'un historien el d'un archéologue, ou encore plus d'un promoteur de projets de développement ${ }^{\text {". }}$. En agronome, Pillot témoigne de sa prégnance sur les esprits des décideurs actuels : « Si les projets de développement de l'irrigation du nord-ouest du Cambodge fleurissent avec autant de persévérance (malgré les échecs patents), n'est-ce pas parce que l'idée s'est subrepticement imposée que Angkor avait réussi son développement sur cette base ? » $(J R C: 63)$.

La critique de la thèse de la cité hydraulique qui a vu le jour à la suite de l'article de van Liere et dont l'ouvrage de Pillot fournit une nouvelle version, se comprend pour une bonne part comme une réaction à l'égard de cette approche «moderniste » de l'histoire agraire à l'époque angkorienne.

L'intérêt porté à la culture du riz flottant et à la riziculture de décrue relève de cette compréhension nouvelle du rapport des anciens Khmers à leur environnement : moins dominé par le principe d'une artificialisation que par celui d'une utilisation des ressources en eau telles que celles-ci s'offraient à eux. Incidemment, la démarche conduit à souligner l'absence de certains savoir-faire - ainsi les remarques de van Liere sur la technologie des barrages -, mais sans qu'il y ait à s'en étonner puisque d'autres solutions techniques, plus adaptées, étaient disponibles ${ }^{10}$.

La thématique de la « nature nourricière » prenant le pas sur celle d'une transformation du milieu à la mesure des progrès techniques, il y a là une sorte de retour de balancier que l'historiographie du Cambodge n'est certainement pas la seule (là encore) à avoir expérimenté. Nous pouvons suivre Pillot dans cette direction, pour autant que cette thématique nouvelle ne soit pas invoquée en contradiction avec toute idée d'une anthropisation. Comme il le rappelle à de nombreuses reprises, il n'y a pas de riziculture possible sans une bonne maîtrise de l'eau et ce contrôle de l'eau impose de façonner le paysage. Cela vaut pour la riziculture de décrue comme pour la riziculture des terrasses, irriguée ou non.

Cela pourrait sembler moins vrai de la culture du riz flottant, mais l'exemple du delta du Mékong montre que les choses sont plus complexes. À partir du XIX ${ }^{\mathrm{e}}$ siècle, comme à époque ancienne, de très nombreux canaux, qui ont requis la mobilisation d'une force de travail considérable, ont été creusés en différentes parties du delta, y compris là où la hauteur de la nappe d'inondation a sans doute déterminé le choix d'une culture du riz flottant (cf. infra). Leur utilité a notamment consisté à faciliter le retrait des eaux en fin d'inondation, en évitant la stagnation trop longue de celles-ci dans les cuvettes à proximité des canaux et, partant, le développement des mauvaises herbes faisant obstacle à la croissance des jeunes pousses du riz ${ }^{11}$.

9. Au Cambodge. on le sait, la liste est augmentée d'un tout autre type d'acteurs : les dirigeants et cadres du régime du Kampuchea démocratique (1975-1979) qui ont poussé jusqu'à l'absurde les principes d'une artificialisation ignorant les "réalités têtues de la topographie » et les savoirs empiriques des paysans (voir à ce sujet les remarques in JRC:101-113).

10. Van Liere $1980: 271,274$; voir la note 13 ci-dessous.

11. Les observations faites sur le réseau moderne livrent bien sûr des pistes d interprétation précieuses pour l'analyse de l'ancien réseau hydraulique creusé dans la plaine de Oc Eo et, plus généralement, dans le delta du Mékong. Un premier bilan de l'étude menée sur ce vaste réseau remontant à l'époque 
L'ampleur des travaux de terrassement n'est pas le plus significatif dans le rapport des hommes à leur environnement ; importent surtout les solutions que ces travaux sont supposés apporter. S'il y a bien toujours anthropisation, il faut néanmoins faire la distinction entre deux modalités différentes de celle-ci : artificialisation en dépit des contraintes du milieu ou, comme le suggère Pillot, mise en valcur selon un mode défini par celles-ci.

\section{Périodiser l'histoire agraire, avec ou sans "révolution verte"}

Nous suivrons avec davantage de réserves l'analyse de Pillot dans sa périodisation de l'histoire agraire. Il a certainement raison de refuser l'idée d'une « révolution verte » à Angkor, tout au moins selon les termes définis par Groslier. On comprend naturellement que c'est l'opposition à la thèse de la " cité hydraulique » qui prime dans le propos de l'auteur.

Si l'époque angkorienne n'apporte en elle-même aucune innovation technique majeure, l'agriculture khmère a-t-elle pour autant traversé les siècles en conservant fondamentalement le même profil, comme le suggère Pillot à l'occasion : " sans changer ni ses techniques ni son mode d'organisation, une population clairsemée a pu ainsi se reproduire et s'étendre en relative autonomie " 12 ? La critique de l'approche "moderniste " de Groslier n'est-elle pas, sur ce point, menée trop loin pour aboutir à une manière de « primitivisme » (mettant ainsi en avant l'inertie d'une agriculture dite « traditionnelle »)? Le débat mérite d'être poursuivi, même s'il faut bien reconnaître que la discussion a été jusqu'à présent assez mal engagée ${ }^{13}$.

Il importe tout d'abord de préciser de quoi on parle. Les outils (charrue, herse, charrette, etc.), comme les savoir-faire de chaque riziculture, peuvent être déjà connus, mais où et par qui ? Quelle est la part des paysans qui, effectivement, disposent de ces outils et mettent en cuvre ces savoir-faire? Dater des innovations ou des emprunts techniques est une chose, la question de leur diffusion, aussi bien dans l'espace que dans la société, en est bien sûr une autre.

du Funan a été présenté au 19" congrès de l'Indo-Pacific Prehistor 1 Association en décembre 2009 (Bourdonneau 2009).

12. JRC: 496. La réflexion sur l'autonomie va ici de pair avec cette autre observation de l'auteur selon laquelle toute fonction utilitaire des baray étant à ses yeux écartée, l'agriculture khmère n'aurait eu recours ni à des aménagements importants, ni à une mise en commun d'investissements coordonnés observation en partie inexacte, comme nous allons y revenir.

13. Voir le traitement sévère réservé à l'article de van Liere par Pottier : "one can easily notice the contradiction between a sentence like: "hydraulic works were severely restricted by the limited technology. of the Khmer" (van Liere 1980. 274) and reality. Simply remembering the huge size of the West baray, it appears that the ancient Khmers were not really restricted. In fact, one could paraphrase this author; considering that "modern hydranlic science severely restricted the understanding of Khmer hydraulic works" "; "after explaining why the Khmers were not able to build sizeable dams, he proceeds to offer some examples of such a Khmer dam large enough to change the course of a river" (Pottier 2000 : 102 103). Quelle que soit la validité des observations et hypothèses avancées par van Liere, son propos, tel qu'il est développé dans l'article cité, ne souffre pas à nos yeux de ces ambiguïtés. La remarque sur la «technologie limitée » des anciens Khmers est explicitée dans les deux phrases qui lui font suite et qu'on ne saurait omettre sans biaiser le sens d'une telle remarque : "they lacked the technique of constructing weir foundations. They also lacked the technique of using clay cores in dams ». Les anciens Khmers ignoraient ces techniques mais leur mise en ouvre, suggère simplement l'article. qui ne se contredit pas sur ce point, n’est pas nécessaire à la construction d'ouvrages de dérivation (ou à celle de vastes bassins). En substance, le propos de van Liere invite à ne pas tout confondre (ouvrages de dérivation ou de « retardement " de l'inondation et ouvrages fonctionnant comme d'authentiques barrages). 
En ce domaine, quand bien même une large part des données nous reste inaccessible, il est sans doute raisonnable d'envisager le schéma d'une diffusion très contrastée des techniques, selon le rythme propre de chaque région - une diffusion probablement compliquée par celle de l'usage du fer. Il faut là considérer l'horizon des principaux acteurs concernés, figurant parmi les plus humbles de la société, à savoir les paysans eux-mêmes. Soit, précisément, celui privilégié par Pillot dans une grande partie de son ouvrage mais que les grandes fresques de son chapitre historique perdent un peu de vue. On y comprend aisément qu'aucune invention matérielle ne peut vraiment avoir de conséquence révolutionnaire sans s'enraciner dans l'intelligence, l'expérience des hommes en prise directe sur la nature, « au ras des rizières »; c'est de leur esprit d'observation et d'initiative que naissent ou renaissent les innovations.

À des fins de périodisation, deux enseignements peuvent être retenus de ces mécanismes d'appropriation. Il apparaît tout d'abord qu'il n'y a guère de supposée " révolution agricole » qui ne se ramène à un usage plus fréquent de techniques connues depuis longtemps. Pillot ne suggère d'ailleurs pas autre chose lorsqu'il définit l'agriculture angkorienne comme une " harmonieuse conjugaison » des trois rizicultures maîtrisées dès l'époque préangkorienne. Une telle lecture invite, certes, à mettre l'accent sur la continuité des pratiques. Mais on voit aussi, et à l'inverse, que les transformations lentes et les innovations infimes ne sont pas sans efficacité et peuvent aboutir à des changements en profondeur ${ }^{14}$. Entre la thèse d'une «agriculture traditionnelle " traversant les siècles et celle de « révolutions » successives, une large place peut ainsi être faite à des approches alternatives, attentives aux transformations mais sur le temps long, sensibles aux innovations mais sans en négliger les plus infimes.

Ajoutons que ce que nous avons rappelé pour les outils et les savoir-faire vaut naturellement pour l'ensemble des facteurs de production : la surface des terres, le nombre des hommes et des bêtes ne furent certainement pas des grandeurs fixes et égales d'une région à l'autre. Pillot ne l'ignore pas lorsqu'il souligne à son tour comment la croissance angkorienne alla probablement de pair avec une extension des surfaces cultivées, en particulier aux dépens de la forêt. L'hypothèse d'une augmentation du nombre des hommes mérite aussi d'être intégrée à la réflexion, sans avoir toutefois à imaginer une "formidable immigration de main-d'œuvre ", comme l'avance un peu imprudemment l'auteur. Nous pouvons également songer au vaste dossier constitué sur la diffusion du riz précoce du Champa, qui rappelle que l'histoire du matériel végétal ne saurait se limiter à l'adoption d'un riz indien ${ }^{15}$.

Nos réserves à l'égard du propos de Pillot tiennent surtout au fait que la périodisation retenue n'intègre guère l'attention portée à la complexité de ces évolutions. Pillot nous semble quelque peu victime de la perspective même de son ouvrage, né du regard porté sur le présent défini comme un moment charnière, marquant le terme d'une évolution démographique au-delà de laquelle « le statu quo n'est plus possible, la transformation $[\ldots]$ indispensable » $(J R C: 496)$. À l'aune des bouleversements majeurs qui s'annoncent et s'esquissent déjà, les transformations anciennes peuvent, de fait, sembler mineures. Mais, à l'évidence, une telle perspective travaille à rebours de la volonté, affichée dans le reste de l'ouvrage, de développer une approche dynamique des pratiques agricoles.

14. Baschet $2006: 132$.

15. Voir notamment Gernet 1999: 281 . 
Nous n'oublions certes pas que Pillot émet lui-même l'hypothèse d'une "révolution verte » : celle-ci est désormais placée à l'aube de la période historique, en invoquant surtout les emprunts faits à l'Inde (selon une conception, nous l'avons dit, quelque peu datée de l'indianisation). Il est vrai que, ce faisant, Pillot ne fait en somme que repousser dc quclques siècles en arrière, par rapport à la thèse de la "cité hydraulique ", le temps des grandes innovations techniques. De ce point de vue, la différence entre l'approche qu'il défend et celle qu'il dénonce n'est pas aussi marquée que ce que nous avons suggéré. En amont des divergences sur la chronologie desdites « révolutions vertes », l'une et l'autre thèse mettent, à nos yeux, le même accent sur les innovations (ou les emprunts) techniques comme source majeure des transformations observées. Si aucune de ces innovations n'est à relever - ainsi que le suggère Pillot pour les deux derniers millénaires -, les pratiques agraires sont alors supposées demeurer fondamentalement les mêmes. Il y a là une manière d'isoler les innovations techniques, pour les ériger en critère dominant, au sein de tout ce qui compose par ailleurs un "mode de production " (pour employer ici un langage d'inspiration marxiste), étant entendu que ce « tout » s'étend bien au-delà des seules « forces productives » évoquées plus haut.

Rappelons, à ce sujet, tout l'apport de la technologie culturelle, qui s'est imposée comme une discipline majeure lors de ces trois dernières décennies : les techniques sont des phénomènes sociaux à part entière dont l'analyse doit faire apparaître leurs relations aux autres pratiques sociales ${ }^{16}$. Les techniques ne forment donc pas un donné qui affecterait de l'extérieur les sociétés et dont il suffirait de dater les avancées pour en déduire une périodisation (où alterneront sans surprise de longues phases de stagnation et de brèves périodes de « révolution »). Périodiser l'histoire agraire, il faut insister sur ce point, impose de prendre la mesure d'un mouvement d'ensemble qui nécessite de combiner, autant que possible (et à bonne distance des formules faciles sur l' "État théocratique » mobilisant les masses autour de ses dieux et de ses princes), histoire économique et histoire sociale, histoire politique et histoire culturelle. Ici comme ailleurs, " tout change d'un même mouvement : les croyances, les pratiques religieuses, les habitudes alimentaires, les cadres de vie, les méthodes de travail, les formes de sociabilité ${ }^{17}$.

\section{L'exemple funanais}

Une rapide évocation de l'exemple préangkorien du Funan nous servira ici d'illustration. Il paraît difficilement envisageable, en effet, de s'interroger sur la vie agricole de la période funanaise sans, dans le même temps, considérer les grands processus qui marquent les premiers siècles de notre ère. C'est avec l'indianisation et la formation de l'État - le Funan peut de fait être défini comme le premier grand État indianisé de la région - une puissante dynamique qui, à l'évidence, se met en place et embrasse tout un ensemble de phénomènes.

Les études manquent, bien sûr, pour prendre la mesure d'une telle dynamique et on n'a d'autre choix que de faire une large place aux hypothèses. Le travail mené sur l'ancien réseau hydraulique de la plaine de Oc Eo (probable capitale méridionale du Funan) est néanmoins riche d'enseignements ${ }^{18}$. Il révèle l'ampleur considérable des aménagements hydrauliques entrepris pour mettre en valeur cette partie du delta transformée en véritable

16. Lemonnier $1983: 11$.

17. Bonnassié $2001: 58$.

18. Bourdonneau 2009. 
mer intérieure lors de la crue annuelle du Mékong. Le Funan, peut-on suggérer, fut ce premier État dont l'histoire, intrinsèquement liée à la conquête du delta, se lit également comme celle d'un « front pionnier " ${ }^{19}$. Il faut alors restituer ce qu'un tel mouvement de conquête suppose comme processus de (re-)structurations, que l'on considère la taille des exploitations, la forme de la propriété, l'organisation de l'habitat ou la morphologie sociale. Quel système de culture était privilégié dans ce contexte? La littérature archéologique a mis l'accent ces dernières années sur le rôle nouveau joué par la culture du riz de décrue ${ }^{20}$. L'hypothèse n'est pas sans susciter certaines réserves, car elle s'accorde assez peu à cette dynamique de front pionnier.

Dans les régions soumises à l'inondation, nous l'avons vu, le riz de décrue ( $d r y$ season flood-recession rice) permet une forte intensification par unité de surface, mais il est très exigeant en travail, notamment parce qu'il nécessite des dispositifs d'exhaure de l'eau. S'il a connu un essor à l'époque moderne, comme le montre bien Pillot, c'est en raison de l'augmentation démographique, de la réduction de la superficie des exploitations et de la commercialisation des motopompes ${ }^{21}$. À l'inverse, la culture du riz flottant est un système de culture extensif qui offre une excellente rémunération du travail permettant une accumulation dès lors que l'on dispose d'une surface importante. Avec un rendement moyen de $2 \mathrm{t} / \mathrm{ha}$, la productivité du riz flottant peut être estimée à 40-45 $\mathrm{kg}$ par jour de travail, " ce qui est considérable », observe Pillot. La culture d'un hectare de riz flottant n'exige pas plus de 50 jours de travail : c'est deux fois moins que le riz de saison sur terrasse et près de trois fois moins que le riz de décrue. Les systèmes à riz flottant offrent ainsi l'avantage de libérer un temps important pour d'autres activités. On peut songer à la chasse ou à la pêche ou à d'autres cultures 22. Mais c'est là surtout un atout important

19. Le concept de «front pionnier 》- défini comme un espace (de conquête) intermédiaire où se constituent et se structurent de nouvelles formes sociales - retient l'attention en ce qu'il permet précisément de lier trois domaines d'analyse : l'organisation spatiale, les formes sociales, l'histoire environnementale. Ce faisant, il souligne ce qui fait la spécificité d’un milieu deltaïque (comme espace social et comme espace naturel). une dialectique de l'ouverture et de la fermeture, le delta étant à la fois cet espace répulsif (que les conditions d’inondation amènent à fuir) et un espace attractif (dont les vastes étendues sont à conquérir). Cf. Leveau 2005. S’agissant du Funan, il amène également à réfléchir sur le rôle d'une dynamique de front pionnier dans celle de la formation de l'État.

20. Cf. Fox et Ledgerwood 1999: 38. La culture de riz de décrue est supposée, selon ces auteurs, avoir soutenu le développement du Funan (l'étude repose sur l'observation des pratiques récentes autour de la région d Angkor Borei). Pour sa part. Pillot reste attentif au rôle joué par la culture du riz flottant : «Dès le troisième siècle. elle fonde les économies émergentes de plusieurs cités du bas-delta du Mékong, dans l'actuel Vietnam » (JRC': 77, avec un renvoi à van Liere 1980).

21. "Avant la diffusion des motopompes au début des années 1990, il fallait ajouter aux charges de travail familial le temps nécessaire aux pompages à la noria [...] qui représentaient de 25 à 35 jours supplémentaires par hectare $\gg(J R C: 304)$.

22. Nous songeons notamment à la culture de la canne à sucre. Celle-ci pourrait fournir la clé de l'un des passages du Jin Shu (ou Ilistoire des Jin) relatif à l'agriculture du Funan : "Ils sèment une année et récoltent pendant trois " (trad. Pelliot 1903:254). Ce passage a suscité de nombreux commentaires, parmi lesquels on relèvera celui de Ronald David Hill. L'auteur relève tout d'abord que le caractère. utilisé par l'auteur du Jin Shu ne désigne pas l'action de « semer », contrairement à ce qu“indique la traduction de Pelliot, mais celle de « planter». Hill n'en maintient pas moins l'hypothèse que c"est bien là une culture du riz qui est décrite et conclut : There is therefore no evidence here of anmally-sown irrigated crops but merely of either a one-year-in-thee rain fed system of rice cultivation with what mal be termed a self-fallow and collection of ratoon crops or: alternativell: of three rears cropping of clearings » (Hill 1977: 16-17). L'hypothèse de "ratoon crops " retient en particulier l'attention, mais il est curieux que Hill ne l'envisage que pour la riziculture : une telle pratique, mise en cuvre précisément sur trois ans, est l'une des caractéristiques distinctives de la cannè à sucre, dont l'exploitation par les paysans funanais est par ailleurs bien attestée par les sources chinoises (ci. Nan Qi shu, trad. Pelliot 
au regard du travail considérable (pour défricher et creuser les canaux) qu'a nécessité la mise en valeur du delta à une période et dans une région où l'on imagine difficilement une abondance de bras.

\section{La quête du surplus}

Au sein du modèle formulé par Pillot, l'accent mis sur la pauvreté des terrasses peut être lu comme une autre manifestation de l'approche teintée de primitivisme qui s'affirme dans les années 1980. La perspective de l'ouvrage est peut-être là aussi en cause; à se préoccuper des réponses à apporter aux défis actuels, l'auteur n'est-il pas davantage enclin à souligner les limites de "l'agriculture traditionnelle», et a fortior i les limites de celle-ci dans la zone des terrasses?

Pillot livre, nous l'avons vu, une description en des termes assez misérabilistes de la condition des familles paysannes dépendantes de la seule riziculture des terrasses : des "régions toujours pauvres », au potentiel de développement agricole réduit, tout juste en situation d'autosuffisance alimentaire. Le contraste avec les surplus dégagés par la riziculture de décrue est particulièrement accusé, de sorte que celle-ci apparaît comme la seule voie effectivement viable pour un État angkorien en expansion 23 . Là encore, il nous semble nécessaire de nuancer le propos de Pillot en modulant différemment l'argumentation développée par l'auteur et en privilégiant les observations qui suivent la partie historique de son ouvrage.

Citons, pour l'heure, une seule de ces observations, avant d'y revenir plus bas. Elle concerne directement la question des rendements. Pillot se fait l'écho dans son chapitre 4 des études menées ces dernières années mesurant précisément les rendements obtenus dans les parcelles, rompant avec la pratique antérieure des estimations obtenues par simple enquête ou dans le cadre d'essais agronomiques : " on s'est aperçu, à cette occasion, que la situation était moins dramatique que ce qui se disait couramment $\gg^{24}$. Certes, subsiste le constat de rendements très variables (d'une province à l'autre ou au sein même d'une province) et qui, pour une part, se cantonnent dans la fourchette basse des résultats obtenus par les autres systèmes de culture. La tendance générale d'une légère réévaluation mérite néanmoins d'être relevée, pour autant que l'on s'entende sur ce que l'on cherche : quel surplus pour quel usage et quelle croissance?

Opposants et partisans de la thèse de la « cité hydraulique » partagent, de ce point de vue également, les mêmes postulats. Quels que soient les auteurs, le point de départ des réflexions demeure le même constat, formulé sur le ton de l'évidence : «la construction des temples d'Angkor, un ensemble architectural gigantesque, qui a dû mobiliser la force de travail de dizaines, voir de centaines de milliers de personnes » $(J R C: 61)$. L'agriculture

1903 : 262). Toujours cultivée à notre époque, la canne à sucre offre l’avantage d’être bien adaptéc aux sols salés et acides du delta du Mćkong. Ajoutons que certaines traces visibles sur les photographies aériennes de plaine de Oc Eo sont susceptibles d'être interprétées comme les indices de l'exploitation de la canne à sucre à époque ancienne (communication personnelle de David Biggs).

23. Nous voyons ainsi que, de manière plus générale, l'accent sur le riz de décrue semble avoir été mis aux dépens de la riziculture de riz flottant pour la période funanaise et de la riziculture des terrasses pour la période « post-funanaise ».

24. «Tichit, pour les années 60, citait des moyennes inférieures à 1 t/ha. Au début des années 1990 . le chiffre de 1 à 1.5 tha était le plus souvent repris dans les rapports d'experts de passage. sans que l'on puisse bien savoir sur quoi il reposait, si ce n'est sur des statistiques agricoles qui sous-évaluaient systématiquement la production $»(J R C: 245)$. 
doit atteindre un niveau élevé de productivité pour permettre de prélever les importants surplus nécessaires à cette formidable entreprise de construction.

Nous renvoyons ici aux réflexions de Paul Veyne et de tant d'autres : "L'inégalité aboutit à un effet de focalisation, à un épiphénomène trompeur; quand une fraction, même réduite, du revenu global de toute une société est concentrée sur un objectif déterminé, parure monumentale ou armement atomique, les résultats paraissent gigantesques à l'échelle individuelle, même si la société en question est paysanne et pauvre " ${ }^{25}$. Il n'y a pas de lien simple entre activité de construction et prospérité, précisément en raison du caractère munificent et ostentatoire des ouvrages d'architecture ou d'urbanisme retenus imprudemment comme indicateur économique. Les réalisations les plus brillantes peuvent se détacher sur fond d'un réel dénuement si la part de la société qui réunit le surplus et détermine son affectation en décide ainsi.

En d'autres termes, toute réflexion sur l'histoire agraire gagne à se tenir à bonne distance des formulations très emphatiques qui accompagnent souvent l'évocation des temples d'Angkor. Que l'on se tourne vers la riziculture des terrasses ou la culture du riz de décrue, rien ne sert de se mettre en quête de surplus tels qu'ils fourniraient la clé d'un « miracle angkorien ». Ce dont il s'agit de rendre compte est, nous l'avons rappelé, la complexité d'un mouvement d'ensemble, non un miracle. Pour cela, il faut travailler à des hypothèses moins fortes, plus légères que celle d'une riziculture de décrue au rendement d'autant plus exceptionnel que la contribution des terrasses serait insignifiante, ou que celle d'une « cité hydraulique » qui permettrait de s'affranchir des contraintes du milieu.

Dans cette perspective, la réévaluation du rendement des parcelles dans la zone des terrasses, aussi minime soit-elle, n'est pas indifférente. Elle est une donnée, parmi bien d'autres, à considérer dans une réflexion qui doit prendre la juste mesure d'une croissance faiblement marquée, entre une vision pessimiste qui néglige le potentiel de productivité des terrasses car jugé d'emblée trop bas et un excès d'optimisme qui spécule inutilement sur d'improbables surplus.

\section{L'essor des terrasses, sans les baray}

Pillot ne manque pas de le rappeler : les terrasses constituent l'essentiel du Cambodge rural d'aujourd'hui, et l'on peut raisonnablement supposer que la remarque s'applique aussi dans une large mesure à l'époque angkorienne (les riz dits de « saison » représentent aujourd'hui, selon les chiffres retenus par Pillot, environ $81 \%$ des surfaces totales en riz et la base économique essentielle de 7,5 millions de personnes) (JRC:232). Comme nous allons y revenir, il ne fait à peu près aucun doute qu'elles ont joué un rôle majeur dans l'économie du Cambodge ancien, sans que l'on ait, en disant cela, à reconduire telle ou telle version de la « cité hydraulique ». De cette relative réhabilitation des terrasses que nous appelons de nos vœux en dehors du sillon tracé par Groslier, le chapitre 4 de Pillot nous fournit de nombreux éléments, à commencer par l'observation sur les rendements à laquelle nous venons de faire allusion. Prises isolément, de telles observations peuvent sembler insignifiantes mais elles dessinent, au final, une vision d'ensemble de la zone des terrasses qui, historiquement, n'est pas dépourvue de dynamisme et de potentialités de développement, y compris lorsqu'il est fait abstraction des apports très récents de la modernisation des technologies. 


\section{"Jouer au plus près" ?}

Dans cette attention nouvelle portée au dynamisme des terrasses, nous reconnaissons l'apport d'une approche menée au plus près de la réalité du terrain et sachant adopter le point de vue des ménages paysans. Dans la mouvance d'une réhabilitation de « l'acteur social » qui dépasse largement les seules études du monde rural, l'accent est désormais mis sur la capacité d'agir et d'innover du paysan cambodgien : son action est interprétée en termes de stratégies mises en œuvre selon de nombreux paramètres, même si la prise en compte de ces derniers n'est pas toujours formulée clairement (et sans devoir être pour autant considérée comme inconsciente). C'est dire que les pratiques observées sont le fruit de choix dotés d'une rationalité propre, selon les opportunités perçues comme telles. Ils révèlent la part de liberté, certes contrainte, qui subsiste dans la conduite des acteurs, une conduite qui ne peut être ramenée, pour cela, à une simple réponse automatique à des stimuli extérieurs ${ }^{26}$.

Il est juste, cependant, de relever que ce type d'approche s'inscrit dans le prolongement de certaines intuitions fondamentales des monographies plus anciennes. Dans son étude fameuse du village de Lovéa, à l'ouest de Siem Reap, Gabrielle Martel faisait observer que la dépendance des facteurs climatiques donnait aux riziculteurs des terrasses une « mentalité de joueurs » : « on tente une mise sur une terre, dans certaines conditions et on gagne ou on perd; dans ce dernier cas, il ne reste qu'à tenter une autre mise. Comme il faut compter avec des phénomènes impossibles à contrôler, beaucoup de villageois se contentent de peu, paraissent jouer au plus près, ils offrent le moins de surface possible aux forces adverses $\gg 27$.

Cette aptitude des riziculteurs à « jouer » sur les caractéristiques de chaque parcelle « jeu » complexe qui intègre celui sur les semences ${ }^{28}$ - est assurément l'un des aspects du potentiel de développement de la zone des terrasses. Elle nous renseigne sur cette capacité d'adaptation et, partant, d'innovation, sur laquelle insiste Pillot. Certes, sous la plume de Martel, elle semble surtout révélatrice des conditions de vie difficiles des riziculteurs.

Insistons bien sur le fait qu'il ne s'agit pas de nier cette précarité des familles. De la même façon, on doutera assez peu que les conditions de vie de ceux qui, à l'époque angkorienne, travaillaient la terre, furent difficiles, tout autant ou même bien davantage qu'aujourd'hui (même si une telle histoire des plus humbles et des liens de dépendances, plus ou moins serviles, qui les attachaient aux plus " grands », pour autant qu'elle nous est accessible, reste elle aussi entièrement à écrire). Il n'existe pas cependant de liens simples entre ces conditions d'existence et le potentiel agricole des terrasses. Les observations de Martel montrent qu'une telle précarité s'accompagne de dispositions d'adaptation remarquables; à suivre Pillot, ce sont ces mêmes dispositions qui ont été mobilisées dans le développement et les innovations de ces dernières années. En reprenant la formule de Diepart, nous dirons que les stratégies mises en œuvre par les paysans comportent un

26. Sur la formulation de ce type d'approche, appliquée au monde rural du Cambodge contemporain, voir aussi Diepart et al. 2005 : 3-4. Par comparaison, les descriptions psychologisantes, brossées à grands traits, du « paysan cambodgien » dans la fameuse somme de Jean Delvert, paraissent aujourd'hui bien désuètes (Delvert 1960: 137 sqq., chap. 5-II. « Les traits du caractère »).

27. Martel $1975: 309$.

28. Prolongeant les remarques de Martel, Pillot souligne la préférence des paysans pour des variétés de semences offrant « une espérance de production relativement stable dans la fourchette des aléas de manque d'eau ou d'excès d'eau et, cela, dans les différents types de parcelles ", et en aucun cas "selon leur capacité à offrir des rendements élevés " (JRC:279-280). Les paysans combinent également, selon les parcelles, des variétés de photosensibilité différentes de façon à répartir les risques de mauvaises récoltes et à étaler les points de travail (ibid. : 233). 
versant «offensif » et « défensif » (Diepart et al. $2005: 3-4)$. Cette double orientation trouve en particulier à s'illustrer dans les « mauvaises années». Intensification des activités de pêche, d'élevage ou de collecte du sucre, diminution des rations alimentaires, mobilisation des réseaux d'alliance, les mesures prises dès les premiers signes d'une disette à venir sont multiples. La sphère agricole est, elle aussi, directement mobilisée : « on cherche [...] à récupérer dès l'année suivante le niveau habituel des réserves tout en rendant de nouveaux services ou en faisant de nouveaux dons à tous ceux qui ont aidé la famille à passer la difficulté. Pour cela, il faut augmenter la production. Si c'est possible, on emprunte des terres, en particulier auprès des veuves que l'on assiste en temps ordinaires, pour augmenter provisoirement la surface cultivée. Le cas échéant, on étend les cultures de "riz de proximité », qui permettent de faire deux cycles successifs sur une même parcelle » (JRC:218-219).

L'éclairage porté sur les stratégies mises en place par les acteurs livre là une utile mise en garde : on aurait tort de percevoir le paysan des terrasses comme courbé et passif sous le joug d'un déterminisme environnemental particulièrement éprouvant et dès lors de n'imaginer d'autre échappatoire que venant de l'extérieur : révolution technologique et/ou réalisation monumentale d'un pouvoir central mobilisant des ressources inédites.

Emblématique à de nombreux égards, la culture du « rí de proximité » retiendra tout particulièrement notre attention, prenant bonne note du traitement privilégié dont elle fait l'objet dans l'ouvrage de Pillot. Celui-ci retrace le parcours singulier de cette riziculture. Riz de cycle court non photopériodique, cultivé à proximité des maisons pendant la première moitié de la saison humide, il était considéré jusqu'à récemment « comme la culture du pauvre, une culture de soudure $»$, pratiquée avant le repiquage du riz de saison, lorsqu'une disette s'annonçait $(J R C: 251)$. Cette même culture, observe Pillot, apparaît aujourd'hui comme l'une des plus prometteuses en termes de développement.

Différents facteurs ont entraîné cette récente évolution : la diffusion de variétés modernes de riz de cycle plus court que les variétés locales; la reconstitution et le développement de l'élevage bovin et porcin, permettant d'augmenter la quantité de matière organique épandue dans les parcelles; la reconstitution d'un marché de riz incitant les paysans à intensifier leur activité. Ce sont là autant de facteurs très spécifiques aux transformations économiques de ces dernières années, difficilement transposables à une époque plus ancienne et qui intéressent donc moins notre propos - même si le fait, nous l'avons dit, qu'une «culture du pauvre » fournisse la base d'une modernisation agricole mérite en soi d'être relevée.

Il est néanmoins d'autres caractéristiques de ce « riz de proximité », plus indépendantes de cette modernisation, qui ont une portée plus grande. C'est, de manière générale, tout ce qu'implique précisément cette " proximité » par laquelle Pillot choisit de nommer ce type de culture. Elle désigne le lien étroit entre les maisons et les rizières où elle est pratiquée. Sont concernées les zones d'habitat diffus, en particulier là où les maisons se distribuent le long des pistes ou des rivières. Dans les parcelles proches des maisons s'accumulent l'eau et la fertilité. Le haut niveau de fertilité organique s'explique par la disponibilité en fumier résultant de «la fréquentation de ces zones par le bétail en saison sèche et les restitutions engendrées par l'activité domestique ». La plus grande maîtrise de l'eau est, elle, rendue indispensable par le calendrier précoce : semés en pépinière dès les premières pluies de mai, les plants de riz doivent recevoir une irrigation complémentaire afin de pallier les insuffisances et l'irrégularité des précipitations à cette période peu avancée dans la saison des pluies. "Pour assurer cette irrigation complémentaire, 
les paysans aménagent individuellement de petites mares au pied de leur maison », ces petites mares étant remplies dès les premières pluies par drainage des champs environnants $(J R C: 250)$. Enfin ce lien de proximité facilite grandement la surveillance des parcelles en une période où, les rizières n'étant pas encore installées, la divagation des bovins est d'autant plus libre $(J R C: 251)$.

Les rendements obtenus varient considérablement selon la fertilité des parcelles mais aussi, et surtout, selon la plus ou moins bonne technicité du paysan qui, insiste Pillot, est ici décisive plus que partout ailleurs au Cambodge. Les plus nettes améliorations de rendement, observe enfin l'auteur, sont dues à une meilleure maîtrise de l'eau : "C C'est la fonction principale [...] de l'équipement des exploitations des terrasses en forages agricoles, là où la nappe est proche ou, dans les autres cas, en mares privées ou collectives » $(J R C: 256)$.

Maîtrise de l'eau (avec des ressources en ligne basse, cf. infira), habileté technique, proximité des maisons et des rizières dans le cadre d'un habitat diffus ou étiré (et source d'une plus grande fertilité), tels sont les éléments saillants qui paraissent riches d'enseignements bien au-delà du cas particulier de la culture du riz de proximité et de la double récolte qu'elle autorise (la culture du « riz de proximité » précédant celle d'un riz de saison). Il n'est évidemment pas indifférent que ces éléments reproduisent pour une part les pratiques spécifiques à la culture des pépinières : "Les paysans apportent toujours un grand soin à leurs pépinières [...]. Dans la mesure du possible, les pépinières sont situées sur un sol fertile, dans des endroits frais, faciles à irriguer, près de la maison, ce qui favorise aussi un bon suivi et un bon entretien ${ }^{29}$.

\section{Se concilier le paysage}

La culture du « riz de proximité » et celle des pépinières relèvent de cette même logique d'ensemble qu'évoque Pillot en conclusion de la partie de son ouvrage consacrée à la riziculture des terrasses et qui lui semble sous-jacente tant aux pratiques traditionnelles qu'aux récentes innovations.

La maîtrise de l'eau, rappelle tout d'abord Pillot, est « une priorité absolue ». Qu'il s'agisse des pépinières ou des parcelles repiquées dans la culture du " riz de proximité », l'insuffisance et l'irrégularité des pluies conduisent les paysans à recourir, autant que possible, à une irrigation complémentaire. Les ressources en eau sont celles, nous l'avons $\mathrm{vu}$, des mares creusées à proximité des maisons. À de nombreuses reprises, l'auteur attire l'attention sur le rôle similaire joué par les réserves d'eau formées dans les canaux creusés lors de la période khmère rouge $(J R C: 250, \mathrm{n} .45)$. De manière générale, ce sont, dans le paysage, l'ensemble des bas-fonds remplis au cours des premières pluies qui sont susceptibles de servir à cette irrigation d'appoint.

L'importance de ces réserves d'eau détermine les limites et les possibilités de développement de la production. La multiplication des mares et leur implantation en plein champ a ainsi beaucoup contribué, observe Pillot, à l'extension récente de la culture du «riz de proximité » et à libérer quelque peu le système de «l'impératif de proximité entre l'habitat et la rizière » $(J R C: 252)$. Mais l'auteur note que d'autres évolutions ont été ici décisives comme la réalisation de forages agricoles et le recours à des motopompes. Pillot

29. JRC : 234. Pillot fait observer par ailleurs que les cultures du « riz de proximité » se trouvent. de façon prévisible, en concurrence avec les pépinières de riz de saison qui traditionnellement occupent les mêmes parcelles $(J R C: 251)$. 
rappelle ailleurs qu'une autre voie de développement de l'irrigation consiste bien sûr à pomper dans les rivières mais c'est aussitôt pour ajouter que « cette possibilité est cependant rare sur les terrasses, contrairement à ce qui existe en zone de berge " (JRC:272).

Au final, le tableau qui est dressé du potentiel des réserves en eau sur les terrasses est, de fait, assez mitigé, a fortiori s'il est fait abstraction de ces évolutions récentes : un réseau hydrographique insuffisant, des mares en nombre limité, de capacité réduite, en raison de leur faibles dimensions (surtout en profondeur) ou encore de leur absence d'entretien et dont la fonction agricole est secondaire ${ }^{30}$. On comprend dès lors la vision assez pessimiste que l'auteur, préoccupé du développement de demain, propose du système de culture «traditionnel » sur les terrasses. On comprend surtout combien, à ses yeux, une telle riziculture ne pouvait guère contribuer à soutenir une croissance économique à époque ancienne.

Or, c'est là, nous semble-t-il, que le propos de Pillot pâtit d'un survol trop rapide des données archéologiques. L'auteur sous-estime l'ampleur des travaux de terrassement (en dehors de ceux déterminés par les baray) réalisés à époque ancienne.

Les cartes archéologiques successives de la région d'Angkor - la plus récente en date étant celle publiée par l'équipe du Greater Angkor Project ${ }^{31}$ - en livrent un premier aperçu. De par leur nature même, elles offrent cependant l'inconvénient de proposer une vision très sélective de l'occupation du sol, retenant du tissu paysager les éléments que leurs auteurs ont jugés les plus pertinents. Il est là indispensable de consulter, d'une part, les photographies aériennes (à commencer par les plus anciennes d'entre elles) et, d'autre part, les images satellites dont certaines, à l'heure de Google Earth (il est vrai que la genèse de l'ouvrage de Pillot y est sans doute antérieure), sont désormais accessibles à tout à chacun avec un remarquable niveau de résolution. Un œil quelque peu exercé y prend sans peine la mesure des efforts accomplis pour modeler les formes du paysage : canaux, fossés, digues, retenues, bassins de toutes dimensions (sans oublier les douves) parsèment le territoire. Dans de nombreux cas, les profils s'étant émoussés, les dépressions s'étant comblées, ces structures apparaissent aujourd'hui comme des formes « fossiles » qui, au mieux, fonctionnent comme ces bas-fonds remplis par les pluies évoqués par Pillot. Leur ancienneté ne fait souvent pas de doute, même s'il faut naturellement restituer une chronologie complexe et sur le temps long.

Ce qui est vrai d'Angkor et de ses environs l'est aussi de nombreuses autres régions. C'est, virtuellement, l'ensemble de la zone des terrasses - là tout au moins où l'on perçoit des traces d'occupation ancienne - qui montre ce même souci de modeler le paysage (selon, certes, des configurations et des densités différentes d'une région à l'autre). Un immense travail de cartographie attend, en ce domaine, les archéologues pour en rendre compte de façon précise.

Plusieurs grands principes paraissent à l'œuvre dans ce travail de la terre qui affecte aussi bien le réseau hydrographique que la topographie.

30. «Plus leur profondeur est importante (et leur surface d’évaporation réduite), plus le stockage qu'elles pourront réaliser sera efficient. Or, elles tendent à se combler » $(J R C: 261)$.

«La première mesure qui s'impose est donc la protection des berges et le curage régulier des réservoirs, voire leur surcreusement $\gg(J R C: 262)$.

31. Evans et al. 2007. 
Modifier le tracé des rivières (ou des bras de rivières plus ou moins pérennes), les détourner ou les ramifier, semble obéir ici à la volonté récurrente d'en rallonger toujours un peu plus le cours ${ }^{32}$. Un second procédé, bien évoqué par van Liere, consiste à barrer les lits des rivières par l'érection de levées de terre, non pour en stopper rigoureusement le cours mais pour, selon les cas, retarder l'inondation ou étendre celle-ci au-delà des limites normales de crue. Le modelage de la topographie répond également au souci de collecter, d'acheminer et de retenir localement les eaux des pluies et du ruissellement, mais aussi celles des cours d'eau et de leur crue, dans les multiples points bas structures en creux artificielles ou (plus ou moins) naturelles, et de profondeur variable : les nombreux bassins, douves et canaux venant s'ajouter, dédoubler ou se superposer aux dépressions d'arrière-berge, aux paléo-chenaux et aux bas-fonds aménagés dont Pillot a souligné le rôle dans l'agriculture traditionnelle.

Nous ne voyons là aucune volonté de s'affranchir réellement des contraintes du milieu et de gommer les contrastes qui induisent de telles contraintes. Si le paysage a été beaucoup plus remanié que ne le suggère Pillot, cette anthropisation se fait selon le mode de mise en valeur défini plus haut : non pas une artificialisation mais un jeu sur ces contrastes qui peut conduire à les accentuer.

Reprenons l'exemple de la topographie. Comme l'écrit Pillot en conclusion de son ouvrage, le Cambodge livre cette première impression d'un pays extrêmement plat, mais la monotonie apparente du paysage est ici trompeuse. Dans des systèmes agraires où la culture se fait en grande partie sous une lame d'eau, quelques dizaines de centimètres de différence peuvent être décisives $(J R C: 495)$. Dès lors, et dans un contexte de rareté de l'eau, tous les points bas de la topographie importent. Là s'achemine et s'accumule l'eau des premières pluies et des ruissellements; là les terres ont toutes les chances d'être plus fertiles. Or, à lire les formes du paysage, nous l'avons vu, les anciens Khmers ont précisément déployé beaucoup de leurs efforts à exploiter, démultiplier et sur-creuser ces bas-fonds, accentuant les effets de cette micro-topographie. Ce travail sur le relief a été réalisé selon un principe de dispersion imposé par le paysage ${ }^{33}$, à l'opposé de la logique qui sous-tend le modèle de la «cité hydraulique » et la thèse d'une irrigation par gravité assurée par la diffusion d'une vaste nappe de parcelle en parcelle. Un tel principe de dispersion va tout autant à l'encontre de la conception d'un réseau de canaux aveugle aux mouvements, infimes mais déterminants, du terrain et dont les ouvrages planifiés par les Khmers Rouges ont offert la caricature.

\section{Un jeu inégal?}

Renoncer à « la cité hydraulique » n'est donc pas renoncer à toute hypothèse sur la maîtrise de l'eau dans la zone des terrasses. Entre la thèse d'un vaste système tripartite de contrôle de l'eau centré sur les baray (collectant, stockant puis redistribuant celle-ci à grande échelle, au long, ou non, d'un réseau de canaux commandés par les baray) et

32. À ce sujet, il n'est pas interdit de songer au motif de la descente de la Gañgā dans la chevelure de Śiva, tel qu'il est évoqué notamment dans l'une des stèles érigées aux angles du baray oriental : "C'est par ce roi des rois [Yaśovarman] qu'a été creusé cet étang [...] pareil à la rivière du ciel qui s'écoule en un flot ralenti, quand elle tombe de la tête du destructeur des trois forteresses, répandue par le mouvement continu de sa danse » (K. 280-D, st. 22, trad. Bergaigne et Barth 1893 : 452).

33. À cette dispersion répond, on le sait, celle des " points hauts », tertres légèrement surélevés et petites éminences sur lesquels sont bâtis, comme autant d'îles dans le paysage, les maisons et les lieux de culte (cf. Ovesen, Trankell et Öjendal 1996, article au titre évocateur : « when everv household is an island $\gg)$. 
celle d'une dépendance exclusive à l'égard des précipitations, nous voyons que de nombreuses hypothèses alternatives peuvent être envisagées. Certes, l'ensemble des stratégies évoquées ci-dessus et dont le paysage conserve la trace, peut paraître encore insuffisant, non seulement au regard des enjeux qui s'offrent au Cambodge d'aujourd'hui (cela va de soi), mais aussi aux yeux de l'historien qui s'interroge sur les ressorts de l'économie angkorienne. La densité de ces aménagements, si elle est plus importante que ne le suppose Pillot, reste bien sûr relative - a fortiori si l'on considère que de telles réalisations se sont étalées sur plusieurs siècles. Il va de soi que de nombreuses parcelles demeuraient isolées de tout point d'eau et relevaient de cette riziculture pluviale dont l'auteur décrit par ailleurs la précarité.

À une telle objection, on répondra tout d'abord, en nous répétant sur ce point, qu'il s'agit de prendre la mesure d'une « croissance infime » et d'appréhender un mouvement d'ensemble dans sa complexité (mieux saisi par Pillot dès lors que son analyse s'ancre dans le monde contemporain). À ce stade bien peu avancé des connaissances, il faut en quelque sorte faire feu de tout bois et multiplier les hypothèses. Une hausse possible de la démographie, l'augmentation progressive des surfaces cultivées - selon le principe d'une " colonisation intérieure » qui conduit à défricher toujours de nouvelles terres ${ }^{34}$-, une plus grande diffusion des techniques, des conditions climatiques éventuellement plus favorables ${ }^{35}$, des rendements du « riz de saison » qui, on l'a vu, sont peut-être à réévaluer (légèrement) ou encore une diversification accrue des activités complémentaires comme le maraîchage, la pêche ou l'exploitation du palmier à sucre : selon une liste non exhaustive, ce sont là autant de propositions, ajoutées à l'attention portée aux aménagements visant à la maîtrise de l'eau, qui autorisent une réhabilitation « mesurée » de la riziculture de terrasse.

Il importe d'ajouter une dernière observation qui prime peut-être sur toutes les autres. Il s'agit de réaffirmer une nouvelle fois la nécessité d'articuler ces réflexions à une authentique sociologie. La recherche d'un équilibre entre une vision pessimiste et une vision optimiste de la contribution des terrasses n'a guère de pertinence sans s'interroger simultanément sur la construction du lien social au sein des campagnes khmères et sur la nature des dynamiques qui en résultent. Dans un contexte de rareté de l'eau (« premier des maux », comme l'écrit Pillot, dans un pays qui est pourtant noyé sous les eaux la moitié de l'année), les ouvrages hydrauliques sont des objets d'enjeux sociaux majeurs. Cela est vrai tout particulièrement des bassins dont la multiplicité des fonctions dit assez la hauteur de ces enjeux - à l'irrigation du maraîchage, des pépinières et des rizières, s'ajoutent l'usage domestique, la pêche, l'abreuvement et l'entretien des animaux ${ }^{36}$.

34. L’hypothèse a été émise de longue date par Mestier du Bourg 1970) (rappelée in Lieberman 2003 : 226). Pillot souligne, pour sa part, l’intérêt des observations faites autrefois par Delvert, selon lesquelles «les paysans préfèrent attaquer la forèt dense que de remettre en culture les zones herbeuses qui avaient été autrefois exploitées : grâce à l'humus accumulé, la fertilité des sols y est vraisemblablement très supérieure » $(J R C: 91)$.

35. Dans quelques pages très synthétiques d’un ouvrage à visée comparatiste, Lieberman porte une attention particulière à l’hypothèse d’un régime des pluies plus favorables (2003: 224-226). Comme pour les autres hypotheses envisagées ici, la démonstration reste encore à faire. Nous renvoyons à ce sujet aux études palynologiques en cours conduites par Dan Penny dans le cadre du Greater Angkor Project.

36. Pillot rappelle également qu'un repiquage d’un riz traditionnel peut être pratiqué à l'intérieur même des bassins (JRC : 222). 
Compétition et coopération au sein du srok et autour des bassins

Ceux qui le peuvent creusent leur propre mare familiale, observe Pillot, les autres doivent dépendre des ouvrages « collectifs ». Les guillemets s'imposent : après de nombreux autres, l'auteur rappelle dans le dernier chapitre de son ouvrage, combicn la notion de "communauté villageoise » reste étrangère à la société khmère et évoque à ce sujet "l'étonnant petit nombre, au sein des villages, d'équipements collectifs de conception et de réalisation endogènes, dont le meilleur exemple est l'aménagement agricole $\gg{ }^{37}$. Ailleurs, il relève combien la gestion de l'eau, après le repiquage, demeure des plus sommaires : « il n'y a pas de souci de gestion coordonnée des besoins, ni des excès : tout le système est conçu techniquement pour faire face au mieux, individuellement, à ces aléas » $(J R C: 241)$. Ici comme ailleurs, et en apparence plus qu'ailleurs, la maîtrise et la gestion de l'eau soulèvent et se heurtent, note Pillot, à de sérieux problèmes organisationnels qui sont sources de conflits.

Le Cambodge documenté par les anthropologues et les spécialistes du développement est dominé par cet idéal d'autonomie dont la formule déjà citée-every households is an island - offre un raccourci parlant. Ledit «village » khmer a été beaucoup perçu comme une réalité insaisissable, un espace amorphe au sein duquel la conduite d'une action collective, la définition des responsabilités nécessaire à la mise en œuvre de celle-ci rencontrent, de fait, de nombreuses difficultés. Cette grande fluidité des rapports sociaux au sein du srok a beaucoup dérouté les observateurs, et il n'y a qu'un pas à franchir pour dresser, sur ce constat, le portrait d'une société bloquée.

S'adossant ici à une riche expérience du terrain, il faut reconnaître à Pillot le mérite d'avoir su proposer une vision plus nuancée sur un sujet complexe où beaucoup reste à dire et à faire. Si, là encore, le propos tenu est avant tout tourné vers les défis du Cambodge contemporain, il rejoint sur plusieurs points les préoccupations de l'historien des périodes anciennes. On s'accordera tout d'abord avec Pillot sur le fait que les problèmes de gestion ne sont au Cambodge, pas plus qu'ailleurs, une fatalité. Les mécanismes des relations de coopération demeurent introuvables dans le srok khmer aussi longtemps qu'ils sont appréhendés en référence aux notions problématiques de "communauté villageoise ». D'autres outils conceptuels doivent être mobilisés.

En première approximation, nous pouvons faire nôtre la formule ramassée de Richard O'Connor : "they compete in principle but cooperate pragmatically " ${ }^{38}$. En d'autres termes, le principe d'autonomie des maisons qui règne au sein du srok n'est pas un idéal d'isolement. Partant d'une réflexion sur l'organisation spatiale de l'habitat, nous nous sommes efforcés ailleurs d'illustrer ce point ${ }^{39}$. Si chaque maison peut être définie comme un « monde en soi », elle est intégrée à une trame naturelle qui joue un double rôle : elle est tout à la fois ce qui préserve l'« insularité » des habitations et les intègre dans une

37. JRC : 474-475 citant une autre étude du Gret (Lemarquis 1992).

38. O'Connor 1995 : 976. Dans le cadre d'une vaste fresque comparatiste menée à l'échelle de l'Asie du Sud-Est (avec les avantages et inconvénients que cela suppose), l'auteur oppose cet ethos des "garden-farmers » du monde khmer à celui des « wet rice specialists», que seraient les Birmans, les Tai et les Vietnamiens. "[who] cooperate in principle but compete out of pragmatism ».

39. Bourdonneau 2007 : 144 (se référant en particulier aux travaux d`Augustin Berque). 
relation dynamique. Nous renvoyons là aux nombreuses observations des ethnologues invitant à définir le niveau vicinal du srok khmer comme « un tissu connectif » ${ }^{40}$.

L'accent mis sur le microcosme et, partant, l'autonomie des maisons peut ainsi aller de pair avec un ethos d'égalitarisme source de conformisme ou d'unanimisme ${ }^{41}$. La contradiction n'est qu'apparente précisément parce que cet idéal d'insularité se manifeste dans le maintien d'une distance elle-même chargée de sens : suffisamment marquée pour signifier la séparation, une telle distance ne l'est toutefois pas assez pour signifier la rupture et l'isolement. Elle est avant tout un « espacement » : un espace de transition qui assure autant l'autonomie de chaque maison que leur mise en relation.

Concrètement, la vie au sein du srok repose sur un réseau de relations interpersonnelles au long duquel se construit et est respectée une communauté de valeurs assurant l'articulation de l'individuel au collectif ${ }^{42}$. Ce sont ces relations concrètes, qui se produisent dans un espace d'interconnaissance, qui composent l'horizon de référence. Les nombreuses méprises sur le caractère amorphe de la société khmère proviennent du fait que cet horizon n'est pas valorisé en lui-même : il est cette trame au sein de laquelle se positionne chaque maison, ce « champ matriciel » dont la neutralité garantit l'insularité de chacune.

De cette construction du lien social, plusieurs éléments sont à retenir pour la compréhension de la mobilisation collective au sein du srok. Bien sûr, nous songeons avant tout à la maîtrise de l'eau et à la construction d'ouvrages hydrauliques.

Pour autant que la cohésion nécessaire à de telles réalisations n'est pas imposée aux dépens de l'idéal d'autonomie des maisons, il n'y a là aucune fatalité, aucun mécanisme de blocage qui ne soit inévitable. La relation dynamique que nous venons d'évoquer est à la fois ce qui permet et ce qui définit les limites de la mobilisation. Cette dernière ne saurait être celle d'un groupe « naturel» - en d'autres termes celle d'une communauté qui préexisterait et survivrait à une telle mobilisation. Elle est l'actualisation ou, disons, la mise en ordre de marche de ce réseau lâche d'unités autonomes se plaçant à cette occasion sous l'autorité d'un «chef » qui n'est, là encore, en rien l'émanation d'un tel groupe. Pillot insiste avec raison sur cet aspect : la mobilisation fonctionne aussi longtemps que « personne n'a à s'investir dans des responsabilités importantes et personne n'est chargé d'une fonction qui le distingue du groupe. Celui qui dispose de la capacité à mobiliser le groupe relève généralement déjà, par nature, d'un autre groupe social » (JRC: 479$)$. Son pouvoir, note ailleurs Pillot, " ne procède pas d'une légitimité qui lui serait conférée par le groupe »; il tient, au contraire, à la reconnaissance d'un statut supérieur auquel l'appartenance aux réseaux de pouvoir qui irriguent le sommet de la société contribue fortement.

Les aperçus synthétiques de O'Connor proposent la même lecture. reformulée dans le cadre de son vaste modèle comparatif entre «garden-farmers » et "wet rice specialists" . Là où, chez les seconds, les positions d'autorité reposent sur le respect de la coutume, O'Connor suggère qu'elles se construisent, chez les premiers, davantage sur l'obéissance

40. L’expression " tissu connectif" » est empruntéc à Fabienne Luco (1998: 184). Voir également Forest 1992 ; Népote 1992 (chap. 1), Ovesen, Trankell et Öjendal 1996.

41. Forest $1992: 87-91$.

42. Ainsi peut-on comprendre d’ailleurs que le srok puisse apparaître comme une cellule close sur elle-même (Forest 1992:86). 
et la contrainte ${ }^{43}$. De cette conception politique des ouvrages collectifs, il résulte que les aménagements hydrauliques réalisés ne sauraient être perçus comme une propriété collective. Il est en effet entendu que le premier bénéficiaire est le détenteur de ce pouvoir auquel a été déléguée, dès lors, la responsabilité de la gestion de tels aménagements. Dans cette perspective, l'aménagement réalisé est d'abord l'expression d'un statut et des relations d'autorité (et de dépendance) que ce statut permet d'imposer. Cependant, de telles relations se doublent, en retour, de relations de protection à l'égard des habitants du srok. Ni bien commun, ni propriété privée à proprement parler, l'ouvrage qui est le fruit de la mobilisation relève d'un usage partagé mais qui est hiérarchisé et respecte le principe d'autonomie dominant ${ }^{44}$.

Plus que tout autre aménagement hydraulique, les bassins-trapeang livrent l'illustration de l'inscription dans le paysage de telles dispositions socioculturelles. À cet égard, comme à de nombreux autres évoqués plus haut, ils sont bien emblématiques d'un mode de vie spécifique au monde khmer. Rappelons avec O'Connor, opposant ici les implications sociales du fonctionnement d'un barrage construit en amont et celui d'un bassin creusé en plat pays, que les ressources en eau de ce dernier bénéficient de façon très inégale aux rizières aux alentours, irriguant naturellement en priorité les parcelles les plus proches et les plus anciennement cultivées - tandis que les eaux d'un barrage profiteront indifféremment aux champs situés en aval ${ }^{45}$.

De l'ensemble de ces observations se dégage un enseignement majeur sur lequel Pillot revient dans la conclusion de son ouvrage et qui va nous ramener à l'examen de la riziculture de terrasse à l'époque angkorienne. Nous avions pris précédemment bonne note du fait que la monotonie apparente du paysage du Cambodge rural masquait une réelle diversité géographique et des pratiques culturales. Dans le prolongement des remarques qui précèdent, il s'agit à présent de réaffirmer la nécessité d'intégrer à l'analyse la diversité sociale du monde rural, une diversité que l'idéal de consensus au sein du srok a conduit parfois à occulter.

43. O'Connor $1995: 977$ et $2004: 28$. Les raccourcis qui accompagnent la formulation à grands traits d'une grille de lecture supposée couvrir l'ensemble de l'Asie du Sud-Est, conduisent l'auteur à accuser certainement plus qu il ne faudrait les contrastes entre les populations mises en regard. Sur de nombreux points, les vues de l'auteur demanderaient à être nuancées et discutées. Il en va ainsi, par exemple, de l'usage problématique qui est fait du concept de big men dans la description des « gardenfarmers ", ou de la description des communautés villagcoises vietnamiennes (rangées parmi les «wet rice specialists ») qui ne s'accorde pas à la vision renouvelée qui en est aujourd'hui proposée (cf. Tessier 2003). Il demeure que les intuitions de l'auteur demeurent tout à fait stimulantes pour notre propos.

44. Voir le témoignage édifiant que livre Pillot sur le refus des paysans de se comporter en co-cxploitants d'un système communautaire : "Si une motopompe collective est installée pour remonter l'eau de la rivière, ils préfèrent se rassembler en petits groupes de voisins désirant l'cau au même moment [...]. Les groupes peuvent changer au gré des arrosages, en fonction des souhaits et des capacités de trésorerie de chacun. La souplesse est ainsi privilégiée par rapport à la rentabilité [...]. La caricature de ce système est celle du paysan individuel venant avec sa bouteille d'essence commander un pompage limité à sa propre parcelle » $(J R C: 274)$. «L'un des moyens les plus simples, note ailleurs Pillot, d'offrir un service d'irrigation et de drainage relativement individualisé aux usagers est de maintenir en eau des canaux en ligne basse [à un niveau topographique inféricur à celui des parcelles] dans lesquels les usagers viennent $s$ approvisionner individuellement $[\ldots]$. Les nombreux aménagements de bas-fonds que l'on trouve partout au Cambodge entrent généralement dans cette catégorie » $(J R C: 463)$. Le principe d'une irrigation en ligne basse est bien sûr à l'opposé de celui d'une irrigation par gravité telle qu'elle a été imaginée par Groslier en aval des baray.

45. O’Connor 1995 : 977. Sur les implications sociales différentes d'une irrigation en ligne basse et d'une irrigation en ligne haute, voir la note précédente. 


\section{Essor économique et causalité sociale}

Le Cambodge, terre de contrastes : la proposition est assurément susceptible de nourrir les réflexions de l'historien sur les périodes anciennes. Il va néanmoins de soi que les relations tissées au sein des microsystèmes sociaux centrés sur les ouvrages hydrauliques ne sont pas restées inchangées au fil des siècles. Précisons à nouveau que c'est là la matière d'une histoire socio-économique qui n'est pas encore écrite et qui devra se concentrer autour des questions suivantes : les relations changeantes entre les différentes formations sociales ont-elles favorisé ou inhibé la hausse de la production agricole des terrasses ? Dans quelle mesure les forces de travail disponibles ont été orientées vers la réalisation d'ouvrages hydrauliques à vocation agricole, et quelles furent alors les modalités d'usage de ces ouvrages?

Pour l'heure, nous nous limiterons à observer que le Cambodge ancien voit la constitution de très grands domaines aux mains des hauts dignitaires du royaume, là où le Cambodge « traditionnel » (c'est-à-dire grosso modo celui des XIX $\mathrm{X}^{\mathrm{C}}$ et $\mathrm{XX}$ ( siècles) se caractérise par l'absence ou la faiblesse de la grande propriété ${ }^{46}$. On gagne ici à prendre ses distances à l'égard de la dichotomie entre pouvoir central et communautés locales, à laquelle les débats sur la " cité hydraulique » se sont parfois réduits. D'un côté, les auteurs critiques à l'égard de la thèse de Groslier ont mis l'accent sur la petite échelle des ouvrages réalisés et la faible implication des grands dignitaires et des souverains ; de l'autre, le modèle de la " cité hydraulique » a attribué d'emblée un rôle considérable à la seule royauté.

Les inscriptions montrent assez clairement que nous devons en réalité rendre compte d'une sorte de « jeu à trois » entre le souverain, les « grands » et ceux, de loin les plus nombreux, qui entrent dans la dépendance plus ou moins marquée des deux figures précédentes ${ }^{47}$. En gardant à l'esprit que l'ampleur des terrassements entrepris par les anciens Khmers n'est pas à sous-estimer, il s'agit là de s'interroger sur l'ancrage des réseaux de pouvoir au cour du monde rural et de prendre la mesure de la domination exercée sur les couches les plus humbles travaillant la terre. À titre d'hypothèse de travail, il est certainement à envisager qu'une telle domination ait été beaucoup plus marquée à époque ancienne que dans le Cambodge « traditionnel ».

Si le corpus épigraphique ne saurait fournir toutes les réponses recherchées, il n'en contient pas moins une masse de données susceptible d'alimenter notre réflexion en ce domaine, mais demeurée relativement vierge d'analyses dans ce sens, jusqu'à aujourd 'hui. Naturellement, la cartographie archéologique, complétée par des fouilles, est, elle aussi, appelée à livrer certains éléments de réponse. Elle permet tout d'abord de documenter assez précisément le nombre et la distribution des ouvrages et, partant, le volume de terres déplacées. Elle doit permettre également d'esquisser une première typologie de ces ouvrages : bassins, dérivations, retenues, etc. Ce faisant, elle est bien en mesure de nous renseigner sur l'usage des aménagements réalisés, étant entendu que tout remodelage du paysage ne saurait impliquer une hausse absolue de la production agricole : d'une part car certains ouvrages peuvent n'avoir aucune fonction agricole et, d'autre part, parce que redessiner le tracé d'une rivière, l'interrompre, en détourner les flots vers un autre cours d'eau peut conduire à privilégier telle étendue de rizières aux dépens de telle autre.

46. Delvert 1960:496-499.

47. Nous empruntons la formule à Testart $2001: 43$. Naturellement. les règles d'un tel jeu sont sensiblement complexes : chacune des figures du triangle n'est pas supposéc conserver la même position relative au cours des siècles et peut se dédoubler, voire se subdiviser en de nombreuses figures différentes. 
Ici comme dans bien d'autres économies anciennes, l'un des ressorts majeurs de l'essor de l'agriculture sur le temps long a pu tenir à un équilibre délicat entre une pression accrue exercée par les dominants sur les « forces productives » (sous la forme, en particulier, de prélèvements) et une plus grande maîtrise des «moyens de production » et des «produits du travail» (si l'on veut employer le langage marxiste) laissée aux populations travaillant directement la terre.

Quoi qu'il en soit, cette prise en compte d'une « causalité sociale » oblige à relativiser la pertinence d'une alternative entre une vision pessimiste et une vision optimiste de l'agriculture des terrasses, reposant sur le seul examen des facteurs de développement desdites « forces productives » et « moyens de productions » (progrès ou diffusion des techniques, augmentation démographique, amélioration climatique, etc.).

Insistons sur le fait qu'il ne s'agit pas simplement de rajouter une entrée supplémentaire à une liste de facteurs déjà existants et dont une analyse systémique se fixerait pour objectif de dégager les interactions (selon des effets de feed-back). Les vertus des analyses systémiques ont beaucoup tenu à la critique des théories mono-causales privilégiées par le passé ${ }^{48}$. Elles offrent néanmoins leurs propres inconvénients. L'effet que peuvent avoir sur les sociétés les grands «stress socio-environnementaux » traditionnellement invoqués n'est pas évidemment en cause. Ce qui fait difficulté est le réductionnisme et la pensée mécaniste sous-jacents à ce type de démarche, quels que soient les efforts faits pour allonger la liste des paramètres retenus ${ }^{49}$. Il importe de rappeler à nouveau que les acteurs exercent un contrôle réflexif sur les conditions dans lesquelles ils " font leur propre histoire » : l'incidence effective de tel ou tel facteur d'évolution «présuppose à chaque fois l'existence d'une motivation précise chez ceux et celles sur qui ces forces "agissent" $"{ }^{50}$. Or la définition de telles motivations ne peut se comprendre sans s'interroger sur la logique globale d'une société, au cœur de la mise en place de nouvelles structures sociales. C'est, en dernière analyse, une telle logique qui est la condition fondamentale de l'essor d'une civilisation, "sa causalité non pas initiale mais englobante ${ }^{51}$.

48. Voir en particulier les travaux de Charles Higham qui revendique, à la suite de Colin Renfrew, une «approche à variables multiples » (a multivariate approach, Higham 2002:292). La démarche vise à identifier le plus grand nombre de variables supposées pertinentes pour l'analyse. Le "système global » formé par la société étudiée est subdivisé en plusieurs sous-systèmes, chacun d'entre eux représentant un domaine différent de l'activité sociale (commerce, techniques agricoles, démographie, etc.), dont il s'agit d’analyser les interactions au cours du temps. Ces interactions sont caractérisées par un phénomène de rétroaction et de «multiplier effect ": les changements ou les innovations se produisant dans l'un des domaines de l'activité socialc entraînent des modifications dans les autres sous-systèmes, qui affectent à leur tour les changements de départ. Parmi les tentatives récentes, au sein de l'historiographie sur la région, voir également Lieberman $2003: 65$.

49. Divers travaux, déjà anciens, comme ceux de J. Friedman et M. Rowlands ont bien souligné ces limites des «multivariate models»: « since they are normally restricted to such abstract categories as population size and density, technological organization, trade, warfare, they are not specific enough to account for the actual transition from one social form to another ». Les auteurs remarquent avec pertinence: "This must necessarily be the case where the models in question contain no social properties. It is the integration of the above categories in a social formation which determines their specific effectivity " (Friedman et Rowlands 1977:267).

50. Giddens $1987: 315$.

51. Nous empruntons ici le raisonnement au remarquable ouvrage de Jérôme Baschet sur la civilisation féodale et l'essor économique de l'occident médiéval (Baschet 2006 : 138). On conviendra avec Victor Lieberman (Lieberman 2003), sans suivre néanmoins celui-ci dans le détail de son analyse, qu'il y a là en effet bien des problématiques communes avec l'histoire ancienne de l'Asie du Sud-Est. 


\section{En guise de conclusion}

De façon assez grossière, avec toutes les réserves qu'impose l'immensité de la tâche qui reste à accomplir, un premier modèle de la production agricole du Cambodge angkorien peut sans doute être esquissé. Au sein d'un tel modèle, le même paradigme d'une complémentarité et/ou d'une opposition entre deux modes d'exploitation du sol, l'un intensif, l'autre extensif, pourrait être décliné à différentes échelles, emboîtées les unes dans les autres.

À l'échelle la plus englobante, cette complémentarité serait illustrée dans la relation mise en avant par Pillot entre une riziculture de décrue conduite de façon intensive en bordure du Tonle Sap et une riziculture de saison sur les terrasses, qui aurait « construit » les cités angkoriennes. À une échelle inférieure, ce serait au sein même des terrasses que nous retrouverions ce schéma duel dans la distinction entre, d'une part, les parcelles les plus proches des maisons et/ou les plus privilégiées dans l'allocation des ressources en eau et, d'autre part, celles les plus distantes et les plus dépendantes de l'eau des pluies. À une échelle encore inférieure, au sein des terres où la maîtrise de l'eau est la mieux assurée, on peut songer alors au couple formé par la parcelle repiquée et la pépinière : cette dernière fait, en tout état de cause, l'objet du plus grand soin de la part des paysans khmers et apparait, au même titre que le verger entourant la maison, comme l'incarnation de cet ethos de garden-farmer évoqué par O'Connor.

Il s'agit, ce faisant, de formuler une série d'oppositions relatives, recouvrant tout à la fois la maîtrise de l'eau, les dimensions des surfaces exploitées et la distance à l'habitat, mais qui puisse être également étendue au statut de la main-d'œuvre et au statut foncier des terres cultivées. Dans la zone des terrasses, nous trouverions ainsi, à une extrémité de l'éventail des pratiques possibles, une mise en valeur intensive du sol (avec le soin qu'elle suppose) portant sur des unités d'exploitation de surfaces réduites, à proximité de l'habitation, bénéficiant de ressources en eau grâce à un remodelage ciblé du paysage et sous une dépendance plus étroite à l'égard des couches dominantes; à une autre extrémité, serait définie une exploitation extensive, portant sur de plus vastes superficies, à plus grande distance de l'habitation, sans irrigation d'appoint et qui ferait davantage vivre les paysans que les maitres. Une telle agriculture ne serait évidemment pas sans rappeler les systèmes infield-outfield, caractérisés précisément par cette opposition du centre intensif et de la périphérie extensive, selon une notion qui a été évoquée à propos des campagnes européennes avant la révolution agricole des temps modernes, mais qui connaît aussi une large application aux systèmes agraires tropicaux.

Esquissée ainsi à grands traits, en s'efforçant de trouver la juste distance à l'égard des propres réflexions de Pillot, ni trop loin, ni trop près, la formulation de ce modèle n'a, répétons-le, d'autre valeur que programmatique. L'objectif est, à nos yeux, de se doter d'un premier outil en mesure de rendre compte de la diversité évoquée plus haut, à la fois sur un plan social et environnemental (sans oublier la dimension régionale de cette diversité). Une telle formulation pourrait être rendue plus complexe en introduisant la notion de « jeu » - suivant en cela certaines intuitions anciennes de Gabrielle Martel - et plus encore en prêtant attention à l'évolution des tensions entre les différentes formations sociales. Une question centrale des travaux à venir sera de déterminer si une telle dynamique a pu favoriser ou, au contraire, entraver une hausse de la production agricole. Nous pouvons admettre a priori - c'est-à-dire en dehors de toute hypothèse sur cette évolution - que, sans avoir fait l'objet d'une « révolution verte ", l'agriculture des terrasses a pu apporter une réelle contribution à la croissance angkorienne (sans 
perdre de vue le caractère certainement mesuré de celle-ci). Cependant, une hypothèse optimiste ou pessimiste sur la contribution effective de cette agriculture n'a de sens in fine qu'à la lueur d'une telle histoire sociale qui ouvre elle-même sur une histoire globale du monde angkorien.

\section{BIBLIOGRAPHIE}

BASCHET, Jérôme

2006 La civilisation féodale. De l'an mil à la colonisation de l'Amérique, Paris, Flammarion.

Bfrgaigne, Abel \& Barth, Auguste

1893 Inscriptions sanscrites de Campā et du Cambodge, Paris, Académie des inscriptions et belles-lettres (Notices et extraits des manuscrits de la Bibliothèque nationale et autres bibliothèques 27/2), p. 181-632.

Bonnassié, Pierre

2001 «Le rapport de l'homme à la terre ou les deux sens du mot culture », in P. Bonnassié, Les sociétés de l'An Mil. Un monde entre deux âges, Bruxelles, De Boeck université (Bibliothèque du Moyen Âge 18), p. 51-66.

BOURDONNEAU, Éric

2007 «Réhabiliter le Funan. Óc Eo ou la première Angkor », BEFEO 94, p. 111-158.

2009 "New research on the ancient hydraulic system around the site of Oc Eo. Canals, 'landscape forms' and 'network of forms' ", communication présentée au $19^{\mathrm{c}}$ congrès de l'Indo-Pacific Prehistory Association (29 nov.5 déc. 2009).

Delvert, Jean

1960 Le paysan cambodgien, Paris, L'Harmattan.

Diepart, Jean-Christophe, Dogot, Thomas, Ly Viboth, Loeung Chanthy, Bora, Kathy

2005 Le monde rural dans la plaine centrale du Cambodge. Analyse comparative à partir de cinq communes, Gembloux, Presses agronomiques de Gembloux.

Evans, Damian, Pottier, Christophe, Fiftcher, Roland, Hensley, Scott, TaPley, Ian,

MILNE, Anthony \& BARBETTI, Michael

2007 «A comprehensive archaeological map of the world's largest pre-industrial settlement complex at Angkor, Cambodia " in Proceedings of the National Academy of Sciences of the United States of America, vol. $104 / 36$, p. 14277 14282 .

Fletcher, Roland, Penny, Dan, Evans, Damian, Pottier, Christophe, Barbetti

Michael, Kummu, Matti, Lustig, Terry \& APSARA

2008a «The water management network of Angkor, Cambodia », Antiquity 82, p. 658-670.

Fletcher Roland, Pottier Christophe, Evans Damian \& Kummu Matti

$2008 \mathrm{~b}$ « The development of the water management system of Angkor: a provisional model », Indo-Pacific Prehistory Association Bulletin 28, p. 57-66. 
Forest, Alain

1992 Le culte des génies protecteurs au Cambodge. Analyse et traduction d'un corpus de textes sur les neak ta, Paris, L'Harmattan.

Fox, Jeff \& LEDGERWOOD, Judy

1999 "Dry-Season Flood-Recession Rice in the Mekong Delta: Two Thousand Years of Sustainablc Agriculturc? ", Asian Perspectives 38/1, p. 37-50.

Friledman, Jonathan \& Rowlands, Michael

1977 "Notes towards an Epigenetic Model of the Evolution of "Civilization" ", in Jonathan Friedman \& Michael J. Rowlands (éds.), The Evolution of Social systems, London, Duckworth, p. 201-276.

Gernet, Jacques

1999 [1972] Le Monde chinois, $4^{\mathbb{e}}$ éd. (revue et corrigée), Paris, Armand Colin.

GrdDENs, Anthony

1987 La constitution de la société, Paris, Presses universitaires de France [éd. anglaise : The constitution of society, Berkeley, University of California Press, 1984].

Groslier, Bernard Philippe

1958 Angkor et le Cambodge au xvl' siècle d'après les sources portugaises et espagnoles (avec la collaboration de Charles R. Boxer), annales du Musée Guimet, Paris, Presses universitaires de France (Bibliothèques d'études 63).

1979 «La cité hydraulique angkorienne : exploitation ou surexploitation du sol ? », BEFEO 66, p. 161-202.

Higham, Charles

2002 Early cultures of Mainland Southeast Asia, Bangkok, River Books.

HıI.., Ronald David

1977 Rice in Malaya, A Study in Historical Geography, Kuala Lumpur, Oxford University Press.

Lemarquis, Bruno

1992 Communautés rurales dans le Cambodge de 1992, Phnom Penh, Gret.

LEMONNIER, Pierre

1983 «L'étude des systèmes techniques, une urgence en technologie culturelle", Techniques et Culture 1, actes de la table ronde «Technologie culturelle ", Ivry (nov. 1982), p. 11-34.

Leveau, Philippe

2005 «Frontières internes dans l'empire romain et écologie d'un delta méditerranéen. Le cas du Rhône ", in Robert Bedon \& Ella Hermon (éds.), Concepts, pratiques et enjeux environnementaux dans l'empire romain, Limoges, Pulim (Caesarodunum 39), p. 103-123.

LIEBERMAN, Victor

2003 Strange Parallels: Southeast Asia in Global Context, c. 800-1830. Volume I: Integration on the Mainland, Cambridge, Cambridge University Press.

VAN LIERE, Willem $\mathbf{J}$.

1980 "Traditional water management in the lower Mekong Basin ", World Archaeology 11/3, p. 265-280. 
Llco, Fabienne

1998 "Pratiques et représentations de l'espace d'un village du site d'Angkor : Srah Srang cheung ", in Actes du Congrès national de la recherche socioculturelle sur le Cambodge (Phnom Penh, 17-19 décembre 1997), université de Phnom Penh, p. 181-192.

MARTEL, Gabrielle

1975 Lovea, village des environs d'Angkor. Aspects démographiques, économiques et sociologiques du monde rural cambodgien dans la province de Siem-Réap, Paris, EFEO (PEFEO 98).

Mestikr du Bourg, Hubert (de)

1970 «La première moitié du XI" siècle au Cambodge : Sūryavarman $\mathrm{I}^{\mathrm{er}}$, sa vie et quelques aspects des institutions à son époque ", Journal asiatique 258, p. 281-314.

NÉPOTE, Jacques

1992 Parenté et organisation sociale dans le Cambodge moderne et contemporain. Quelques aspects et quelques applications du modèle les régissant, Genève, Olizane.

O'CONNOR, Richard A.

1995 "Agricultural Change and Ethnic Succession in Southeast Asian States: A case for Regional Anthropology ", The Journal of Asian Studies 54/4, p. 968996.

2004 "Rice in the making of Southeast Asia ", Education about Asia 9/3, p. 27-29.

Ovesen, Jan, Trankell, Ing-Britt \& ÖJendal, Joakim

1996 When Every Household is an Island. Social Organisation and Power Structures in Rural Cambodia (Uppsala Research Reports in Cultural Anthropology 15), Uppsala et Stockholm, Department of Cultural Anthropology of Uppsala University et Swedish International Development Cooperation Agency.

Philiot, Paul

1903 « Le Fou-nan », BEFEO 3, p. 248-303.

Pottier, Christophe

2000 " Some evidence of an inter-relationship between hydraulic features and rice field patterns at Angkor during ancient times ", Journal of Sophia Asian Studies 18, p 99-120.

TESSIER, Olivier

2003 «Fondateurs, ancêtres et migrants : mobilité et reformulation des espaces d'appartenance dans un village du nord du Vietnam », Moussons 6, p. 99-132.

TESTART, Alain

2001 «L'esclavage comme institution », in Alain Testart, L'esclave, la dette et le powvoir: Études de sociologie comparative, Paris, Errance, p. 19-47.

VeYne, Paul

1976 Le Pain et le Cirque. Sociologie historique d'un pluralisme politique, Paris, Seuil. 